\title{
Divergent trajectories of antiviral memory after SARS-Cov-2 infection
}

\section{Adriana Tomic* ( $\nabla$ adriana.tomic@paediatrics.ox.ac.uk)}

Oxford Vaccine Group, Department of Paediatrics, University of Oxford, Oxford, UK https://orcid.org/0000-0001-9885-3535

\section{Donal T. Skelly*}

Peter Medawar Building for Pathogen Research, Nuffield Department of Medicine, University of Oxford, UK https://orcid.org/0000-0002-2426-3097

\section{Ane Ogbe*}

Peter Medawar Building for Pathogen Research, Nuffield Department of Medicine, University of Oxford, UK https://orcid.org/0000-0001-7774-7215

\section{Daniel O'Connor*}

Oxford Vaccine Group, Department of Paediatrics, University of Oxford, Oxford, UK https://orcid.org/0000-0002-6902-9886

\section{Matthew Pace}

Peter Medawar Building for Pathogen Research, Nuffield Department of Medicine, University of Oxford, UK https://orcid.org/0000-0002-5703-3984

\section{Emily Adland}

Peter Medawar Building for Pathogen Research, Nuffield Department of Medicine, University of Oxford, UK

\section{Frances Alexander}

Public Health England, Porton Down, UK

\section{Mohammad Ali}

Peter Medawar Building for Pathogen Research, Nuffield Dept. of Clinical Medicine, University of Oxford, UK https://orcid.org/0000-0003-0170-7182

\section{Kirk Allott}

Department of Clinical Biochemistry, Oxford University Hospitals NHS Foundation Trust, Oxford, UK

\section{Azim Ansari}

Peter Medawar Building for Pathogen Research, Nuffield Department of Medicine, University of Oxford, UK https://orcid.org/0000-0003-2790-8353

\section{Sandra Belij-Rammerstorfer}

Jenner Institute, Nuffield Department of Medicine, University of Oxford, Oxford, UK https://orcid.org/0000-0002-8637-3785

\section{Sagida Bibi}


Oxford Vaccine Group, Department of Paediatrics, University of Oxford, Oxford, UK https://orcid.org/0000-0002-0855-2737

\section{Luke Blackwell}

Oxford Vaccine Group, Department of Paediatrics, University of Oxford, Oxford, UK

\section{Anthony Brown}

Peter Medawar Building for Pathogen Research, Nuffield Department of Medicine, University of Oxford, UK

\section{Helen Brown}

Peter Medawar Building for Pathogen Research, Nuffield Department of Medicine, University of Oxford, UK

\section{Breeze Cavell}

Public Health England, Porton Down, UK https://orcid.org/0000-0001-9897-1590

\section{Elizabeth A. Clutterbuck}

Oxford Vaccine Group, Department of Paediatrics, University of Oxford, Oxford, UK https://orcid.org/0000-0003-1701-1390

\section{Thushan I de Silva}

The Florey Institute for Host-Pathogen Interactions and Department of Infection, Immunity and Cardiovascular Disease, Medical School, University of Sheffield, UK

\section{David Eyre}

Oxford University Hospitals NHS Foundation Trust, Oxford, UK

\section{Amy Flaxman}

Jenner Institute, Nuffield Department of Medicine, University of Oxford, Oxford, UK https://orcid.org/0000-0001-6460-1372

\section{James Grist}

Department of Physiology, Anatomy and Genetics, University of Oxford, Oxford, UK

\section{Carl-Philipp Hackstein}

Peter Medawar Building for Pathogen Research, Nuffield Dept. of Clinical Medicine, University of Oxford, UK https://orcid.org/0000-0002-0821-5204

\section{Rachel Halkerston}

Public Health England, Porton Down, UK https://orcid.org/0000-0001-6420-3331

\section{Adam C. Harding}

James \& Lillian Martin Centre, Sir William Dunn School of Pathology, University of Oxford, Oxford, UK https://orcid.org/0000-0003-1479-959X

\section{Jennifer Hill}

Oxford Vaccine Group, Department of Paediatrics, University of Oxford, Oxford, UK https://orcid.org/0000-0001-6205-7764

\section{Tim James}

Department of Clinical Biochemistry, Oxford University Hospitals NHS Foundation Trust, Oxford, UK

\section{Cecilia Jay}


Peter Medawar Building for Pathogen Research, Nuffield Dept. of Clinical Medicine, University of Oxford, UK

\section{Síle A. Johnson}

Peter Medawar Building for Pathogen Research, Nuffield Dept. of Clinical Medicine, University of Oxford, UK https://orcid.org/0000-0002-4100-8522

\section{Barbara Kronsteiner}

Peter Medawar Building for Pathogen Research, Nuffield Dept. of Clinical Medicine, University of Oxford, UK

\section{Yolanda Lie}

Monogram Biosciences LabCorp, San Francisco, CA, USA

\section{Aline Linder}

Oxford Vaccine Group, Department of Paediatrics, University of Oxford, Oxford, UK

\section{Stephanie Longet}

Public Health England, Porton Down, UK https://orcid.org/0000-0001-5026-431X

\section{Spyridoula Marinou}

Oxford Vaccine Group, Department of Paediatrics, University of Oxford, Oxford, UK

\section{Philippa C. Matthews}

Oxford University Hospitals NHS Foundation Trust, Oxford, UK

\section{Jack Mellors}

Public Health England, Porton Down, UK

\section{Christos Petropoulos}

Monogram Biosciences LabCorp, San Francisco, CA, USA

\section{Patpong Rongkard}

Peter Medawar Building for Pathogen Research, Nuffield Dept. of Clinical Medicine, University of Oxford, UK

\section{Cynthia Sedik}

Monogram Biosciences LabCorp, San Francisco, CA, USA

\section{Laura Silva-Reyes}

Oxford Vaccine Group, Department of Paediatrics, University of Oxford, Oxford, UK

\section{Holly Smith}

Jenner Institute, Nuffield Department of Medicine, University of Oxford, Oxford, UK

\section{Lisa Stockdale}

Oxford Vaccine Group, Department of Paediatrics, University of Oxford, Oxford, UK

\section{Stephen Taylor}

Public Health England, Porton Down, UK https://orcid.org/0000-0002-9936-8539

\section{Stephen Thomas}

Public Health England, Porton Down, UK https://orcid.org/0000-0003-2368-8058

\section{Timothy Tipoe}


Peter Medawar Building for Pathogen Research, Nuffield Dept. of Clinical Medicine, University of Oxford, UK

\section{Lance Turtle}

HPRU in Emerging and Zoonotic Infections, Institute of Infection, Veterinary and Ecological Sciences, University of Liverpool, Liverpool, UK

\section{Vinicius Adriano Vieira}

Peter Medawar Building for Pathogen Research, Department of Paediatrics, University of Oxford, Oxford, UK https://orcid.org/0000-0002-4901-0424

\section{Terri Wrin}

Monogram Biosciences LabCorp, San Francisco, CA, USA

\section{OPTIC Clinical Group}

PITCH Study Group

C-MORE Group

\section{Andrew J. Pollard}

Oxford Vaccine Group, Department of Paediatrics, University of Oxford, Oxford, UK https://orcid.org/0000-0001-7361-719X

\section{Teresa Lambe}

Jenner Institute, Nuffield Department of Medicine, University of Oxford, Oxford, UK

\section{Christopher P. Conlon}

Nuffield Department of Medicine, University of Oxford, Oxford, UK

\section{Katie Jeffery}

Oxford University Hospitals NHS Foundation Trust, Oxford, UK https://orcid.org/0000-0002-6506-2689

\section{Simon Travis}

Oxford University Hospitals NHS Foundation Trust, Oxford, UK

\section{Philip J. Goulder}

Peter Medawar Building for Pathogen Research, Department of Paediatrics, University of Oxford, Oxford, UK

\section{John Frater}

Peter Medawar Building for Pathogen Research, Nuffield Department of Medicine, University of Oxford, UK

\section{Alexander J. Mentzer}

Oxford University Hospitals NHS Foundation Trust, Oxford, UK https://orcid.org/0000-0002-4502-2209

\section{Lizzie Stafford}

Nuffield Department of Medicine, University of Oxford, Oxford, UK https://orcid.org/0000-0002-16105136

\section{Miles W. Carroll}

Public Health England, Porton Down, UK

\section{William S. James}


11. James \& Lillian Martin Centre, Sir William Dunn School of Pathology, University of Oxford, Oxford, UK https://orcid.org/0000-0002-2506-1198

\section{Paul Klenerman\#}

Peter Medawar Building for Pathogen Research, Nuffield Department of Medicine, University of Oxford, UK https://orcid.org/0000-0003-4307-9161

\section{Eleanor Barnes\# ( $\nabla$ ellie.barnes@ndm.ox.ac.uk)}

Peter Medawar Building for Pathogen Research, Nuffield Department of Medicine, University of Oxford, UK https://orcid.org/0000-0002-0860-0831

\section{Christina Dold\#}

Oxford Vaccine Group, Department of Paediatrics, University of Oxford, Oxford, UK https://orcid.org/0000-0003-2314-507X

\section{Susanna J. Dunachie\#}

Peter Medawar Building for Pathogen Research, Nuffield Department of Medicine, University of Oxford, UK https://orcid.org/0000-0001-5665-6293

\section{Research Article}

Keywords: SARS-CoV-2, T cells, B cells, Antibodies, Durability, Healthcare workers, Longevity, variants of concern, COVID-19

Posted Date: June 15th, 2021

DOl: https://doi.org/10.21203/rs.3.rs-612205/v1

License: (c) (i) This work is licensed under a Creative Commons Attribution 4.0 International License. Read Full License

Version of Record: A version of this preprint was published at Nature Communications on March 10th, 2022. See the published version at https://doi.org/10.1038/s41467-022-28898-1. 
Adriana Tomic ${ }^{1 *}$, Donal T. Skelly, ${ }^{2,3,4 *}$, Ane Ogbe ${ }^{2 *}$, Daniel O'Connor ${ }^{1^{*}}$, Matthew Pace ${ }^{2}$, Emily Adland ${ }^{2}$, 4 Frances Alexander ${ }^{5}$, Mohammad Ali ${ }^{2}$, Kirk Allott ${ }^{6}$, M. Azim Ansari², Sandra Belij-Rammerstorfer ${ }^{7}$, Sagida Bibi', Luke Blackwell ${ }^{1}$, Anthony Brown ${ }^{2}$, Helen Brown ${ }^{2}$, Breeze Cavell ${ }^{5}$, Elizabeth A. Clutterbuck ${ }^{1}$, Thushan de Silva ${ }^{8}$, David Eyre ${ }^{3,9}$, Amy Flaxman $^{7}$, James Grist ${ }^{10}$, Carl-Philipp Hackstein², Rachel Halkerston ${ }^{5}$, Adam C. Harding ${ }^{11}$, Jennifer Hill1,12, Tim James ${ }^{6}$, Cecilia Jay², Síle A. Johnson²,3,13, Barbara Kronsteiner ${ }^{2,14}$, Yolanda Lie ${ }^{15}$, Aline Linder ${ }^{1,12}$, Stephanie Longet ${ }^{5,16}$, Spyridoula Marinou ${ }^{1,12}$, 9 Philippa C. Matthews ${ }^{2,3,12}$, Jack Mellors ${ }^{5}$, Christos Petropoulos ${ }^{15}$, Patpong Rongkard², Cynthia Sedik ${ }^{15}$, Laura Silva-Reyes ${ }^{1,12}$, Holly Smith7, Lisa Stockdale ${ }^{1,12}$, Stephen Taylor ${ }^{5}$, Stephen Thomas ${ }^{5}$, Timothy Tipoe $^{2}$, Lance Turtle ${ }^{17,18}$, Vinicius Adriano Vieira ${ }^{19}$, Terri Wrin ${ }^{15}$, OPTIC Clinical Group, PITCH Study Group, C-MORE Group, Andrew J. Pollard ${ }^{1,12}$, Teresa Lambe ${ }^{7}$, Chris P. Conlon ${ }^{20}$, Katie Jeffery ${ }^{3}$, Simon Travis $^{3,21}$, Philip Goulder ${ }^{19}$, John Frater ${ }^{2,3}$, Alex J. Mentzer ${ }^{3,16}$, Lizzie Stafford ${ }^{20}$, Miles W. Carroll ${ }^{5,16}$, William S. James ${ }^{11}$, Paul Klenerman"2,312\#, Eleanor Barnes $2,3,12 \#$, Christina Dold ${ }^{1,12 \#, ~ S u s a n n a ~ J . ~}$

* These authors contributed equally

\# These authors jointly supervised this work and contributed equally

Corresponding authors: Eleanor Barnes, email: ellie.barnes@ndm.ox.ac.uk and Adriana Tomic, email: info@adrianatomic.com

\section{Affiliation}

1. Oxford Vaccine Group, Department of Paediatrics, University of Oxford, UK

2. Peter Medawar Building for Pathogen Research, Nuffield Dept. of Clinical Medicine, University of Oxford, UK

3. Oxford University Hospitals NHS Foundation Trust, Oxford, UK

4. Nuffield Dept of Clinical Neuroscience, University of Oxford, UK

5. Public Health England, Porton Down, England

6. Department of Clinical Biochemistry, Oxford University Hospitals NHS Foundation Trust, Oxford, UK

7. Jenner Institute, University of Oxford, UK

8. The Florey Institute for Host-Pathogen Interactions and Department of Infection, Immunity and Cardiovascular Disease, Medical School, University of Sheffield, UK

9. Big Data Institute, Nuffield Dept. of Population Health, University of Oxford, UK

10. Department of Physiology, Anatomy, and Genetics, University of Oxford, UK

11. James \& Lillian Martin Centre, Sir William Dunn School of Pathology, University of Oxford, Oxford, UK

12. NIHR Oxford Biomedical Research Centre, Oxford, UK

13. Oxford University Medical School, Medical Sciences Division, University of Oxford, Oxford, UK

14. Oxford Centre For Global Health Research, Nuffield Dept. of Clinical Medicine, University of Oxford, UK

15. Monogram Biosciences LabCorp, San Francisco, CA, USA

16. Wellcome Centre for Human Genetics, University of Oxford, UK

17. HPRU in Emerging and Zoonotic Infections, Institute of Infection, Veterinary and Ecological Sciences, University of Liverpool, UK

18. Tropical and Infectious Disease Unit, Liverpool University Hospitals NHS Foundation Trust (a member of Liverpool Health Partners), Liverpool, UK

19. Peter Medawar Building for Pathogen Research, Department of Paediatrics, University of Oxford, Oxford, UK 
20. Nuffield Department of Medicine, University of Oxford, Oxford, UK

21. Translational Gastroenterology Unit, Nuffield Department of Medicine, University of Oxford, Oxford, UK

22. Mahidol-Oxford Tropical Medicine Research Unit, Bangkok, Thailand

\section{Acknowledgments:}

The authors wish to thank all the healthcare worker volunteers who participated in this study, and Suki Kenth for administrative support.

\section{Funding statements}

This work was funded by the UK Department of Health and Social Care as part of the PITCH (Protective Immunity from T cells to Covid-19 in Health workers) Consortium, with contributions from UKRI/NIHR through the UK Coronavirus Immunology Consortium (UK-CIC) and from the Huo Family Foundation.

AT is supported by the EU's Horizon2020 Marie Sklodowska-Curie Fellowship (FluPRINT, grant number 796636). DS is supported by the NIHR Academic Clinical Fellow programme in Oxford. MAA is supported by a Wellcome Trust Sir Henry Dale Fellowship (220171/Z/20/Z). DWE is a Robertson Foundation Fellow. PCM is funded by a Wellcome intermediate fellowship, ref. 110110/Z/15/Z. LT is supported by the Wellcome Trust (grant number 205228/Z/16/Z) and the National Institute for Health Research Health Protection Research Unit (NIHR HPRU) in Emerging and Zoonotic Infections (NIHR200907) at University of Liverpool in partnership with Public Health England (PHE), in collaboration with Liverpool School of Tropical Medicine and the University of Oxford. PK and EB are NIHR Senior Investigators and PK is funded by WT109965MA. SJD is funded by an NIHR Global Research Professorship (NIHR300791).

The views expressed are those of the author(s) and not necessarily those of the NHS, the NIHR, the Department of Health and Social Care or Public Health England.

\section{Contributions}

EB, PK, CD and SJD conceptualised the study. SJD, PK, EB, CPC, DTS and LS designed and oversaw the clinical study. LS, DE, KJ, PM, AJM, PG, SAJ, ST, the OPTIC Study Group and the C-MORE Group contributed to the implementation of the clinical study. AO, AT, CD, DOC, DTS, MP, MWC, WSJ, EB, PK, and SJD designed and oversaw the laboratory studies. AJP, AO, CD, EA, JF, PJG, MWC, WSJ, $E B, P K, S J D$ and $T L$ were responsible for the implementation of the laboratory testing, while $A B, A C H$, $A F, A L, B C, B K, C H, C J, C P, C S, E A C, F A, H B, H S, J G, J G-J, J H, J M, K A$, LB, LS-R, LSt, LT, MAA, MA, MLK, PR, RH, SB, SB-R, SL, SM, ST, STh, TJ, TT, TW, VAV, YL were responsible for laboratory testing and assay development. AT and DOC undertook the advanced data analysis. AO, AT, CD, DOC, DTS, EB, PK, and SJD prepared the manuscript, which was reviewed by all contributing authors. All other authors contributed to the implementation of the study and data collection. 


\section{Competing Interests}

93 DWE declares lecture fees from Gilead, outside the submitted work. No other competing interests 94 declared.

95

96 Key words: SARS-CoV-2, T cells, B cells, Antibodies, Durability, Healthcare workers, Longevity, 97 variants of concern, COVID-19

98

99 Abstract

100 Severe acute respiratory syndrome coronavirus 2 (SARS-CoV-2) infection is normally controlled by 101 effective host immunity including innate, humoral and cellular responses. However, the trajectories and 102 correlates of acquired immunity, and the capacity of memory responses months after infection to 103 neutralise variants of concern - which has important public health implications - is not fully understood. 104 To address this, we studied a cohort of 78 UK healthcare workers who presented in April to June 2020 105 with symptomatic PCR-confirmed infection or who tested positive during an asymptomatic screening 106 programme and tracked virus-specific $B$ and $T$ cell responses longitudinally at 5-6 time points each over 1076 months, prior to vaccination. We observed a highly variable range of responses, some of which - T 108 cell interferon-gamma (IFN- $y$ ) ELISpot, N-specific antibody waned over time across the cohort, while 109 others (spike-specific antibody, B cell memory ELISpot) were stable. In such cohorts, antiviral antibody 110 has been linked to protection against re-infection. We used integrative analysis and a machine-learning 111 approach (SIMON - Sequential Iterative Modeling Over Night) to explore this heterogeneity and to 112 identify predictors of sustained immune responses. Hierarchical clustering defined a group of high and 113 low antibody responders, which showed stability over time regardless of clinical presentation. These 114 antibody responses correlated with IFN-y ELISpot measures of $\mathrm{T}$ cell immunity and represent a 115 subgroup of patients with a robust trajectory for longer term immunity. Importantly, this immune116 phenotype associates with higher levels of neutralising antibodies not only against the infecting 117 (Victoria) strain but also against variants B.1.1.7 (alpha) and B.1.351 (beta). Overall memory responses 118 to SARS-CoV-2 show distinct trajectories following early priming, that may define subsequent protection 119 against infection and severe disease from novel variants. 
121 Severe acute respiratory syndrome coronavirus 2 (SARS-CoV-2), an RNA virus that causes

122 coronavirus disease 2019 (COVID-19), first emerged in humans in December 2019 and has since

123 spread globally, with more than 3.56 million deaths reported world-wide (June 2021

124 https://coronavirus.jhu.edu/map.html). Although the majority of infections cause asymptomatic or mild 125 disease, a significant minority develop a severe illness, requiring hospitalisation, oxygen support, and 126 invasive ventilation ${ }^{1}$. Healthcare workers (HCW) have been at the forefront of caring for patients with 127 SARS-CoV-2 infection in community and hospital environments during the pandemic. High exposure 128 rates have meant that a significant proportion of HCW have become infected and HCW most commonly 129 infected are those working on the front line in patient facing roles, predominantly in acute medical 130 specialities ${ }^{2}$. Older age, comorbidities and male sex remain the dominant factors that predispose to 131 severe outcomes ${ }^{3}$ - since HCW are predominantly younger and female ${ }^{2}$, most have developed mild 132 disease, although deaths are widely reported in this population.

134 Starting early in the pandemic, we and others have sought to characterise the immune responses during 135 SARS-CoV-2 infection that are associated with viral clearance and disease severity. SARS-CoV-2 136 infection has been associated with the generation of high magnitude, broad $\mathrm{T}$ cell responses and high 137 titres of immunoglobulin G ( $\mathrm{lgG}$ ) targeting SARS-CoV-2 spike and nucleoprotein (NP) antigens, 138 particularly in severe COVID-19 ${ }^{4}$. Asymptomatic infection, that appears more common in younger 139 people, may be associated with discordant $T$ cell and humoral immunity with both the absence of $\lg G$ 140 seroconversion in the presence of detectable $T$ cell responses 5,6 or conversely the presence of $\lg$ in 141 the absence of $\mathrm{T}$ cell immune responses ${ }^{7}$. However, more recently critical questions have emerged 142 that include the durability of immune responses following initial infection, the quality of these responses, 143 immune correlates of protection from re-infection, and the capacity of these responses to neutralise 144 new variants of concern (VOC) that have emerged globally. These questions have become paramount 145 following the development of effective vaccines for COVID-19, since deployment of these has been 146 limited by vaccine supply, concerns around adverse events and vaccine hesitancy. Furthermore, to 147 manage limited vaccine resource, people with previous infection are now being offered a single vaccine 148 dose 6 months after infection in many European countries (France, Germany, Spain, and Italy) ${ }^{8}$, on 149 the assumption that natural immunity will protect from re-infection.

150

151 An in depth understanding of immune responses after SARS-CoV-2 infection, and how these change 152 over time, will be critical to understanding who is susceptible to re-infection and to inform vaccine 153 strategies. Currently, the precise correlates of immune protection from subsequent infection after 154 primary disease, or after vaccination, are unknown. Previous reports suggest SARS-CoV-2 IgG 155 antibodies ${ }^{9}$ and previous exposure to seasonal coronaviruses (CoV) ${ }^{10}$ are protective against 156 subsequent SARS-CoV-2 infection. However, since the magnitude of T and B cell responses correlate 157 with each other ${ }^{11}$, dissecting the role of these immune subsets in protection from re-infection or severe 158 disease on re-exposure is challenging. Several groups have now reported that SARS-CoV-2 specific T 159 and $B$ cells decline after acute disease $12,13,14,15,16$, but there is high heterogeneity between individuals 
160 in the levels of measurable immunity in different compartments it is unclear how or if the kinetics of this

161 decline correlate with protection from subsequent infection. Concerns have been raised that SARS-

162 CoV-2 re-infection associated with waning immunity is plausible, particularly since the seasonal

163 coronaviruses, closely related to SARS-CoV-2, commonly re-infect the same host 17, 18. However,

164 waning of immune responses following acute infection, or vaccination is well recognised as part of the

165 normal evolution of memory responses, and reports describing decline in immune responses have

166 focused on ex vivo responses that may not reflect the memory recall potential of viral specific $T$ and $B$

167 cells responses. A particular concern is the identification of SARS-CoV-2 variants of concern (VOC)

168 (B.1.1.7 - alpha, B.1.351 - beta, P.1 - gamma and B.1.617.2 - delta), with mutations which are

169 associated with an increase in transmissibility, severity or escape from vaccine or SARS-CoV-2-induced

170 immunity $19,20,21,22,23,24,25,26,27$. Immune escape, with a failure to neutralise the VOC, in live viral assays

171 in vitro, appear following vaccination and after SARS-CoV-2 infection, and is pronounced in the context

172 of lower antibody titres measured against the initial pandemic strain (B/Victoria).

173

174 Since April 2020, we have followed a cohort of SARS-CoV-2 infected HCW prospectively over time at

175 Oxford University Hospital NHS Foundation Trust. Seventy-eight HCW infected during the UK's "first

176 wave" (defined by positive PCR and seropositive for anti-spike antibodies) were assessed at up to six

177 timepoints and followed for six months in 2020, pre-vaccination, with multiple immune parameters

178 evaluated in more than 430 blood draws. Our aims are to characterise memory $T$ and $B$ cell responses

179 following infection, and to determine the interactions between clinical presentation and the generation

180 and maintenance of $T$ and $B$ cell responses over time. We assess the association of exposure to

181 seasonal coronaviruses and symptomatic SARS-CoV-2 disease with the durability of SARS-CoV-2

182 specific responses. We evaluate the predictive value of clinical and immune parameters measured early

183 after infection on the durability of immune responses using an integrative analysis with a machine

184 learning platform (SIMON) ${ }^{28,29}$. Using this approach, we define a group of high and low antibody responders with a differential capacity to neutralise the VOC.

186 
188 Detailed description of methods are included in the Appendix. HCW volunteer recruitment and ethics

191 We sampled seventy-eight HCW at five or six time points each, over six months. HCWs were recruited from Oxford University Hospitals NHS Foundation Trust after a positive SARS-CoV-2 PCR test ${ }^{2}$ in April-May 2020, including 66 volunteers with symptomatic disease (fever, shortness of breath, cough, loss of taste or smell, sore throat, coryza or diarrhoea) and 12 asymptomatic HCW who did not report any symptoms of COVID-19 in 2020 prior to staff screening or in the seven days following testing positive. The age, sex and ethnicity of the HCW are shown in Supplementary Table 1. Blood samples were acquired at multiple timepoints over 6 months (acute[range:1-20], 28 days [21-41], 56 days [4273], 90 days [74-104], 120 days [110-140], and 180[160-200]) from onset of symptoms in the symptomatic group and from the date of positive PCR test for asymptomatic people diagnosed on screening. Nine hospitalised patients with severe disease were included for comparative analysis. All subjects were seropositive for anti-spike IgG antibodies by ELISA. Mild and asymptomatic participants were recruited under ethics approved by the research ethics committee (REC) at Yorkshire \& The Humber - Sheffield (GI Biobank Study 16/YH/0247). Participants with severe disease were recruited after consenting into either the CMORE study protocol (research ethics committee (REC): Northwest Preston, REC reference 20/NW/0235) and / or Sepsis Immunomics protocol [Oxford Research Ethics Committee C, reference 19/SC/0296]). The study was conducted according to the principles of the Declaration of Helsinki (2008) and the International Conference on Harmonization (ICH) Good Clinical Practice (GCP) guidelines. Written informed consent was obtained for all participants enrolled in the study.

210

Isolation of peripheral blood mononuclear cells (PBMC), plasma and serum

212 PBMCs and plasma were isolated by density gradient centrifugation from blood collected in EDTA 213 tubes, and serum was collected in a serum-separating tube (SST, Becton Dickinson) as previously described ${ }^{5}$ and detailed in the Appendix.

\section{T cell assays}

217 T cell assays including interferon-gamma (IFN- $\mathrm{Y}$ ) Enzyme-Linked immunospot (ELISpot) assay, 7-day 218 proliferation assay and intracellular staining were performed ${ }^{5}$. For IFN-ץ ELISpot assay we used SARS219 CoV-2 peptide pools panning Spike (S1 and S2), membrane (M), nucleocapsid protein (NP), the X220 domain of non-structural protein 3 (NSP3B), open reading frames 3 and 8 (ORF3 and ORF8), and cytomegalovirus, Epstein-Barr virus and Flu peptide pools (CEF) (2ug/ml per peptide) in a 16-18hour incubation at $37^{\circ} \mathrm{C}$. ELISpot plates were read using an AID ELISpot Reader (v.4.0) and results were reported as spot-forming units (SFU) $/ 10^{6}$ PBMC. T cell proliferation assay was performed using fresh or cryopreserved PBMC and CellTrace $\AA^{\circledR}$ Violet (CTV, Life Technologies) labelling and stimulated with peptide pools from SARS-CoV-2 spanning Spike (S1 and S2), M, NP, ORF3 and ORF8, and FEC-T $(1 \mu \mathrm{g} / \mathrm{ml}$ per peptide). On day 7 , cells were stained with fluorochrome-conjugated primary human- 
specific antibodies for CD3, CD4 and CD8 for analysis on a MACSQuant 10 flow cytometer. For

228 Intracellular cytokine staining, PBMC were stained for CD3, CD4, CD8, CD154, IFN- $\gamma$, IL-2 and TNF- $\alpha$ then analysed on a BD LSR II.

\section{Antibody and $B$ cell assays}

232 Standardised total anti-spike IgG ELISA ${ }^{30}$ and anti-spike subclass and isotype ELISAs ${ }^{31,32}$ were

233 performed. A multiplexed MSD immunoassay (MSD, Rockville, MD) was used to measure the IgG responses to SARS-CoV-2, severe acute respiratory syndrome coronavirus-1 (SARS-CoV-1), MERSCoV and seasonal CoVs (human coronavirus (HCoV)-OC43, HcoV-HKU1, HcoV-229E, HcoV-NL63). For Microneutralisation Assay (MNA), the viral isolates used are described in the Appendix, and the assay was performed to determine the concentration of antibody that produces a $50 \%$ reduction in infectious focus-forming units of authentic SARS-CoV-2 in Vero CCL81 cells. Infectious foci were enumerated by ELISpot reader and data were analysed using four-parameter logistic regression (Hill equation) in GraphPad Prism 8.3. The Monogram Bioscience pseudotype neutralisation assay (PseudoNA) was performed ${ }^{30}$.

For the Spike-specific SARS-CoV-2, OC43, HKU1, 229E and $\mathrm{NL} 63 \operatorname{lgG}^{+}$and $\lg \mathrm{A}^{+} \mathrm{B}$ cell memory

244 ELISpot assays, PBMCs were cultured for 3-3.5 days with polyclonal stimulation, and added to Mabtech flurospot plates coated with the relevant spike glycoprotein (SARS-CoV-2 at $10 \mu \mathrm{g} / \mathrm{ml}$, OC43 at $10 \mu \mathrm{g} / \mathrm{ml}$, $\mathrm{NL63}$ at $15 \mu \mathrm{g} / \mathrm{ml}, \mathrm{HKU} 1$ at $5 \mu \mathrm{g} / \mathrm{ml}$ and $229 \mathrm{E}$ at $10 \mu \mathrm{g} / \mathrm{ml}$, all diluted in PBS). All cells were incubated for $\geq 16$ hours at $37^{\circ} \mathrm{C}$, and following development Spot forming units were enumerated using AID ELISpot 8.0 software on the AID ELR08IFL reader. For antibody-dependent effector functions, the spike-specific antibody-dependent effector functions, natural killer cell activity (ADNKA), neutrophil phagocytosis (ADNP) and monocyte phagocytosis (ADMP) were performed ${ }^{31}$, and are detailed in the Appendix alongside the Antibody-dependent complement deposition (ADCD) assay.

252

\section{Integrative analysis using unsupervised and supervised machine learning in SIMON}

254 The integrative analysis was performed using SIMON (Sequential Iterative Modeling "Over Night") software ${ }^{28,29}$ as detailed in the Appendix. The integrated dataset was generated using the standard extract-transform-load (ETL) procedure to merge total of $29 \mathrm{csv}$ files across 14 assays and clinical data via donor-specific variable (Donor ID) according to the SIMON method. The outcome of immune response durability was calculated based on the titre of the anti-N specific antibodies measured 6 months post symptoms onset (pso), and individuals with anti-N antibody titre $\geq 1.4$ were labelled as high responders, while individuals having anti-N antibody titre below 1.4 were low responders. Before integrative analyses, data was pre-processed (centre/scale), missing values were median imputed, features with zero-variance, near-zero-variance and with correlation (cut-off 0.85 ) were removed using SIMON software. The t-distributed stochastic neighbour embedding (t-SNE) (2,000 iterations, perplexity 30 , and theta 0.5 ) followed by clustering (seed number 1337, number of clusters 3 ) was performed to analyse the pre-processed integrated dataset (excluding disease severity and timepoint which are used 
267 immunological parameters (continuous variables, excluding features with less than $10 \%$ of unique

268 values and grouping variable - disease severity). Pairwise correlations of immunological parameters in 269 the integrated dataset were visualized as a correlogram and Spearman's rank correlation coefficient 270 was computed. Values shown on the correlogram were adjusted for multiple testing using Benjamini271 Hochberg correction at the significance threshold (False discover rate, FDR < 0.05). Agglomerative 272 hierarchical clustering was performed on the samples with immunological parameters analysed on day 27328 pso and visualized as the dendrogram on heatmap (tightest cluster ordered first). To identify early 274 immunological signature at day 28 pso that can predict if the individual will be high or low responder 6 275 months pso, we performed SIMON analysis on all immunological parameters (day 28 pso) using 172 276 ML algorithms. Missing values (29\% missingness) were removed using multi-set interaction function 277 ('mulset', SIMON software), resulting in 30 resamples. Each resample was split into train/test partition $278(75 \% / 25 \%)$ preserving the balanced distribution of the outcome class (seed number 1337). The models 279 were evaluated using 10-fold cross-validation on training sets (train AUROC), and additionally on the 280 held-out test sets (test AUROC). The best performing model was built using the Sparse Partial Least 281 Squares (sPLS) algorithm (train AUROC: 0.95 (CI 0.5-1) and test AUROC: 1). In the final step, SIMON 282 calculated the contribution of each feature to the model as variable importance score (scaled to 283 maximum value of 100 ).

284

\section{Statistical analyses}

286 Statistical analysis was performed using R (https://www.r-project.org/), integrative analysis was 287 performed using SIMON software ${ }^{28,29}$, figures were made with $R$ using $R$ package ggplot2 ${ }^{33}$ and 288 GraphPad Prism 8. Kruskal-Wallis test —unless otherwise specified - was used for comparison of the disease severity groups. Wilcoxon rank-sum test —unless otherwise specified - was employed to compare between study time points. A generalised additive mixed model (GAMM) by restricted maximum likelihood (REML) was used to fit the immunological measures (log10 transformed) using Gaussian process smooth term (R package gamm4 ${ }^{34}$ ). ICS cytokine expression analyses was performed using PESTEL v2.0 and SPICE v6.0. Statistical significance was set at $P<0.05$ and all tests were 2-tailed. Machine learning analysis was performed using SIMON software (https://genular.org).

\section{Data Availability}

297 Data relating to the findings of this study are available from a research data repository Zenodo (https://zenodo.org/record/4905965).

\section{Results}

Anti-N IgG decline over time and stratify by disease severity, whilst Anti Spike IgG and memory responses are maintained

303 Anti-nucleocapsid (NP) and spike (S) total $\lg$ ( $\operatorname{tg} \mathrm{lg})$ responses were assessed by ELISA in both symptomatic and asymptomatic individuals (Fig. 1A). The magnitude of the IgG response varied markedly between people in both cohorts, with a proportion of individuals' anti-nucleocapsid tlgG level recorded in the negative or indeterminate range of the assay at all time-points. 
308 Asymptomatic and mild infection induces similar anti-NP responses in the early phase $(<20$ days post 309 PCR positivity/symptom onset) of observed infection ( $P=0.6125$, Supplementary Fig. 1A). However, 310 anti-NP tlgG levels in the two disease cohorts separated as higher levels were observed in those with 311 mild infection from the day 28 timepoint onwards ( $P=0.0015$ for day 28 comparison, Supplementary

312 Fig. 1A). Anti-NP IgG responses waned over time with a significant decrease from approximately day 31328 to day 180 timepoints ( $P=0.00071$ for asymptomatic and $P=7.2 \times 10^{-9}$ for mild symptomatic individuals, 314 Fig. 1A). Most (91.7\%) asymptomatic individuals have an indeterminate or negative anti-NP tlgG response to the nucleocapsid antigen at the day 180 timepoint.

317 Over the time course of observation, anti-spike IgG antibody levels (Fig. 1B) in individuals remained 318 consistent in individuals with asymptomatic $(P=0.35)$ and severe $(P=0.44)$ COVID-19 disease. Similarly, 319 the initial anti-spike tlgG responses increased in individuals with mild disease and remained consistent 320 from day 28 to the 6-month timepoint $(P=0.12)$. Furthermore, disease severity was not a significant 321 predictor of anti-spike tIgG levels in those with asymptomatic and mild SARS-CoV-2 infection throughout the 6-month observation ( $P=0.632$, GAMM, Fig. 1B).

324 In line with the $\operatorname{tgG}$ antibody binding to spike remaining consistent, we observed a steady number of 325 IgG+ memory B cells following an initial increase (Fig. 1C). Anti-SARS-CoV-2 spike-specific IgG+ 326 memory $B$ cells at 6 months following symptom onset were higher than observed during early infection 327 in mild ( $P=0.00042$, Fig. 1C) and severe $(P=0.0027$, Fig. 1C) individuals. For asymptomatic individuals, 328 no change was observed in cell frequencies when comparing the earliest samples collected and 6329 month timepoints $(P=0.54)$, although we note that the timing of infection onset for asymptomatic 330 individuals cannot be precisely determined. Asymptomatic and mild disease did not predict different 331 kinetics for the IgG memory response ( $P=0.284$, GAMM, Fig. 1C).

332

\section{Pseudo-neutralisating antibodies decreased in all disease severities over time}

334 Pseudo-neutralisating antibodies (pseudoNA) were measured in all individuals (Fig. 1D) using an assay 335 that incorporates the spike glycoprotein. Disease severity was a significant predictor of pseudoNA 336 ( $P=0.00073$, GAMM, Fig. 1D) - with higher pseudoNA levels with increasing disease severity at all time 337 points measured (Fig. 1D and Supplementary Fig. 1D). Regardless of disease severity, the pseudo338 neutralising capacity of circulating antibodies to the Wuhan/B lineage virus decreased over 6 months 339 following the detection of SARS-CoV-2 infection (asymptomatic $P=0.023$; mild $P=4.2 \times 10^{-9}$; severe $340 P=0.01$, Fig. 1D). People with severe infection maintained pseudoNA 6 months post symptom onset, 341 and at higher levels than in those with mild or asymptomatic infection ( $P=0.00022$, Kruskal-Wallis test,

342 Supplementary Fig. 1D). The decline was less marked in asymptomatic individuals with no decrease 343 observed from day 28 to day 180 ( $P=0.41$, Fig. 1D); however, the difference in the pseudoNA titres in 344 the mild vs asymptomatic groups remained until day $180(P=0.0148)$. At day 180 post symptom onset 345 or PCR confirmation, one asymptomatic and four symptomatic individuals no longer mounted a positive 
result in the pseudoNA assay, one of whom consistently did not mount pseudoNA capacity at all time points measured.

Mild infection induces a more multifunctional antibody profile

350 A cohort of 30 individuals with mild infection, along with the 9 and 12 participants with severe and asymptomatic infection respectively were selected to comprehensively characterise antibody profiles.

352

\section{Circulating isotypes and subclasses}

Circulating IgM levels decreased over time in those with asymptomatic $(P=0.021$, day $<20$ vs day 180$)$, mild $(P=0.0004$, day $<20$ vs day 180$)$ and severe $(P=0.007$, day $<20$ vs day 180$)$ infection, while IgA levels in participants remained constant in all disease cohorts (asymptomatic: $P=0.65$; mild: $P=0.59$; severe: $P=0.065$ ), throughout the observed 6-month time course (Fig. 2A and 2B) as previously reported ${ }^{12}$. The quantified amounts of $\lg \mathrm{G} 1$ were consistent over time in asymptomatic $(P=0.86$, day $<20$ vs day 180$)$ and severe $(P=0.92$, day $<20$ vs day 180$)$ infection. Despite initial low titres of IgG1 in participants with mild infection, IgG1 circulating antibody titres were maintained from day 28 to 6 months post symptom onset ( $P=0.89$, Fig. $2 \mathrm{C}$ ). While circulating IgG3 antibodies in participants with mild infection were maintained at consistent levels throughout the 6 -month period $(P=0.062)$, levels decreased over this time in asymptomatic ( $P=0.0022$, day $<20$ vs day 180$)$ and severe $(P=0.021$, day $<20$ vs day 180 ) individuals (Fig. 2D). Notable SARS-CoV-2 spike-specific IgG2 responses were only detected at one or more time-points in a small number of individuals tested (asymptomatic: $3 / 12$; mild: 3/30; severe: 1/8) (Supplementary Fig. 2B), while there was no spike-specific IgG4 detected above the LLOQ of the ELISA (data not shown). For all IgG subclasses detected, asymptomatic or mild disease severity were not significant predictors of responses over time (IgG1: $P=0.36$; $\lg G 2$ : $P=0.92$; IgG3: $P=0.0519$, GAMM, Figs. 2C-D). All paired analysis was by Wilcoxon rank sum test.

Diversity of antibody responses

372 We measured the ability of the anti-spike antibodies in those with severe or asymptomatic infection as

373 well as a selection of individuals with mild infection, to induce innate effector functions: ADNP, ADMP,

374 ADNKA and ADCD.

Asymptomatic and mild disease severity was not a significant predictor of Fc-mediated effector functional responses (ADNKA $P=0.798$; ADMP $P=0.117$; ADNP $P=0.206$ ) except for ADCD $(P=0.00314)$ (Fig. 2E-H). Furthermore, normalised ADMP and ADNP scores, as well as the percentage of CD107a-expressing NK cells were stable over time, between 28 days and 180 days post symptom onset or PCR confirmation for those with asymptomatic (ADMP: $P=0.96$; ADNP: $P=0.48$; ADNKA: $P=0.2$ ) and mild (ADMP: $P=0.64$; ADNP: $P=0.75$; ADNKA: $P=0.8$ ) infection (Fig. 2E-H). Similarly, no decline was observed for these Fc-mediated functions from the acute sampling to 6 months post symptom onset in the severe cohort (ADMP: $P=0.89$; ADNP: increase $P=0.021$; ADNKA: $P=0.075$ ) with the ADNP increasing over time $(P=0.021)$ (Fig. 2E-H). ADCD waned dramatically in those with severe disease over the 6 -month period $(P=0.00031)$ but similarly to the other Fc-mediated functions, 
ADCD remained consistent from day 28 to day 180 in asymptomatic $(P=0.34)$ and mild $(P=0.1)$ infection

(Fig. 2E-H). Despite waning over time, ADCD responses differed amongst the disease severity groups out until day 180 ( $P=0.0032$, Kruskal-Wallis test, Supplementary Fig. 1L). All paired analysis were by Wilcoxon rank sum test.

390

We visualised the relative contribution of each of the anti-SARS-CoV-2 spike antibody feature in Fig.

21. The polar plots demonstrate the diversity of asymptomatic and mild infection-induced antibody characteristics and functions on day 28 and day 180. Each wedge represents an antibody feature, and the size of each wedge is indicative of the magnitude of the response. The consistently high spikespecific lgG and spike-specific lgG+ memory B cells is clearly reflected in these plots for both mild and asymptomatic individuals. For both day 28 and day 180, a more multifunctional response was observed in individuals with mild infection, particularly for the antibody-dependent phagocytosis effector functions, which contribute markedly less to the antibody profile of asymptomatic individuals. Over time, few marked changes were observed in the relative contribution of the SARS-COV-2-specific antibody features in asymptomatic individuals, apart from an increased contribution of IgG1 and ADNKA, and decreased IgG3. Similarly, for individuals with mild infection, substantial relative decreases in IgM, pseudo-neutralising antibodies, IgA and IgG3 were noted, as well as relative increases in ADNKA and ADNP to the antibody profile.

404

SARS-CoV-2 infection elicits transient cross-reactive antibodies and memory B cells specific 406 for other circulating coronaviruses.

407 Next, we evaluated the IgG responses to seasonal coronaviruses (229E, HKU-1, NL63-S and OC43-

408 S) severe acute respiratory syndrome (SARS-CoV-1) spike protein and Middle East Respiratory 409 Syndrome (MERS) virus spike protein using the MSD assay (Fig. 3A). IgG responses to these viral 410 antigens were detected at the earliest time points. The kinetics of these $\lg G$ responses followed those 411 seen to SARS-CoV-2 spike, suggesting that seasonal coronavirus cross-reactive responses were 412 enhanced by SARS-CoV-2 infection. Responses to OC43-S, 229-E and HKU-1 were particularly high 413 and correlated significantly with disease severity at day 180 and at the earliest time point assessed (day $414<20$ ) (Supplementary Fig. 2C). The MSD assay also measured IgG responses against SARS-COV-2 415 Spike, NP and the RBD antigens, supporting our observations using the ELISA assay (Supplementary 416 Fig. 2D).

417

418 IgG+ Memory B cells specific for the spike glycoprotein from seasonal coronaviruses (229E, HKU1, 419 NL63 and OC43) were determined at the earliest timepoint available (acute <day 20 or day 28) and the 420 6-month final sampling (Fig. 3B). The lowest responses were observed in 229E and NL63 spike IgG+ 421 ASCs following polyclonal stimulation, which also were consistent over time with the exception of the 422 decreased number of NL63 spike-specific $\mathrm{lg} G+$ memory B cells in individuals with mild infection $423(P=0.0046)$. Higher responses were detected when testing the specificity of cultured PBMCs to the 424 beta-coronaviruses (HKU1 and OC43) spike glycoprotein. However, the boosted memory response 
was transient, particularly in individuals with mild infection (HKU1: $P=1 \times 10^{-7}$; OC43: $P=1.5 \times 10^{-7}$ ) in which the decrease was more marked, which may be due to a higher sample number.

\section{Effector poly-specific SARS-CoV-2 T cells are higher in those with mild symptoms and decline} 6 months after infection

430 We examined the magnitude of the T cell response to SARS-CoV-2 using an ex vivo IFN-y ELISpot assay at 28 days, $90-120$ days and 180 days after SARS-CoV-2 infection ( $\mathrm{N}=64-78 \mathrm{HCW} /$ timepoint, 57 participants at all timepoints (including 12 with asymptomatic infection), and 6 volunteers with severe COVID-19 at day 180 (Fig. 4A and 4B and Supplementary Table 3). We have previously shown that this assay is specific for SARS-CoV-2, with negligible responses detected in SARS-CoV-2 prepandemic unexposed participants 5 .

436

437 IFN-y responses to at least one antigenic pool were seen in $67 / 70(96 \%)$ volunteers tested 28 days after SARS-CoV-2, with a median total response across the pools of 373 (IQR 201-842) SFC/106 PBMC; here a response to spike (S1 and S2) was seen in 61/70 tested (87\%) median 180 (IQR 71364) SFC/106 PBMC, for M in 47/70 (67\%) median 63 (IQR 25-160) SFC/106 PBMC and for NP in 62/70 (89\%) median 121 (IQR 73-250) SFC/106 PBMC. However, total summed responses declined by a median of $60 \%$ after 90 days, and by $75 \%$ at 180 days (Supplementary Table 3). The majority (61/77 $(79 \%))$ of participants had detectable responses to at least one antigenic pool at 180 days, with responses to NP antigen most commonly observed $47 / 77$ (61\%) median 40 (IQR 23-73) SFC/106 PBMC. Responses to ORF3, ORF8 and NSP3B were less frequent than responses to S1, S2, M and NP at day 28 and lower at day 180.

447

IFN-y ELISpot responses to SARS-CoV-2 antigens were higher in the mild symptomatic cohort $(n=66)$, compared to the asymptomatic group $(n=12)$ at 28 days, with median responses to all summed pools 455 (IQR 252-976) SFC/106 PBMC for mild disease compared to 196 (IQR 74-243) SFC/106 PBMC in the asymptomatic group (Supplementary Fig. 3A). There was no significant change in the magnitude of the $T$ cell response in the asymptomatic group in the 6 months after infection (Fig. 4A).

We next used ICS to examine the duration of multiple T cell functions and the polyfunctionality of the T cell response over time at 28 and 180 days pso in individuals with ex vivo T cell ELISpot levels $>100$ $\mathrm{SFC} / 10^{6}$ PBMC for sensitivity reasons $(n=18$ with $n=15$ available at both timepoints for paired analysis (Gating strategy in Supplementary Fig. 3D, results in Supplementary Fig. 4 and Supplementary Fig.5). Similar to the ELISpot data, the majority of T cell responses decreased over time. In terms of functionality, we found that CD4+ T cells were polyfunctional, with the majority of cells expressing $>1$ and up to all 5 functional markers at both timepoints. Similarly, NSP3B-specific CD8+ T cells were also polyfunctional at both timepoints examined, with most cells expressing $>1$ functional marker (Supplementary Fig. 4J). There were no functional changes between the two timepoints. 
We and others have found the assessment of $T$ cell proliferation to be a sensitive method of detecting antigen-specific recall responses. We used this assay to evaluate the frequency of circulating SARSCoV-2-specific CD4+ and CD8+ T cell in our longitudinal cohort $(n=54-57$; gating strategy presented in Supplementary Fig. 3B).

We did not observe any differences in the magnitude of circulating FEC-specific (control) CD4+ or CD8+ T cells within the 6 months period (Supplementary Fig. 3C). In the asymptomatic group, at 28 days pso $7 / 8(87.5 \%)$ made a CD4+ T cell response to at least one SARS-CoV-2 protein (excluding S1 and S2 where have previously reported finding responses in the majority of unexposed volunteers ${ }^{5}$ ) while $5 / 8(62.5 \%)$ of them had CD8 T cell response to at least one of M, NP, ORF3 or ORF8 proteins (Fig. 5A-C Supplementary Table 4). Most of this response was targeted to $M$ and NP (Fig. 5A-C and Supplementary Table 4). At 180 days pso, 6/8 (75\%) of recovered subjects had a CD4+ or CD8+ T cell response which was mostly focused on M, NP and ORF3. We observed no difference in the proliferative capacity of SARS-CoV-2-specific CD4 and CD8 T cells at 28- and 180-days post disease onset in the group with asymptomatic disease $(n=8)$ (Fig. 5A-C and Supplementary table 4 and 5 ).

In the cohort with mild disease, at 28 days, T cell responses to at least one SARS-CoV-2 protein outside of spike region were observed in $42 / 49(86 \%)$ for CD4+ T cells and $45 / 49(91 \%)$ for CD8+ T cells. Similar to the asymptomatic cohort, these responses were focused on M, NP and ORF3 regions of SARS-CoV-2 (Fig. 5A-C, Supplementary Table 4). At 180 days after symptom onset, this frequency of people responding to at least one protein as above reduced to $37 / 49$ (75\%) within CD4+ T cells and 35/49 (71\%) for CD8+ T cells with a focus on M, NP and ORF3 similar to CD4+ T cells (Fig. 5A-C and supplementary Table 4 and 5). In the volunteers with mild disease, we found a significant reduction in the circulating frequencies of SARS-CoV-2-specific CD4+ and CD8+ T cells to all proteins except NP and ORF8 for CD4+ and ORF3 and ORF8 for CD8+ T cells by day 180 (Fig. 5A-C).

When we assessed the difference in the magnitude of the proliferative CD4+ and CD8+ $T$ cell responses at 28- and 180 days pso in both asymptomatic and mild cases (analysed together as one group), we found significantly higher frequencies of SARS-CoV-2 specific CD4+ T cells compared to CD8+ responses at both timepoints in all proteins except NP and ORF8 for 28- and 180-days post symptom onset and ORF3 responses at 28 days post symptom onset only. Our data shows that the bias in antigen-specific responses to SARS-CoV-2 towards CD4+ T cells is maintained in the T cell memory compartment long after recovery from acute infection. Taken together, the results show that at 6 months post infection with SARS-CoV-2, convalescent subjects show diminished but detectable anti-SARSCoV-2-specific memory T cells in both the CD4 and CD8 T cell compartments, with only 8/56 (14\%) showing no proliferative response to any non-spike protein, suggesting durable immune response at least up to 6 months post initial infection.

\section{Integrative analysis to Identify immune and clinical parameters associated with disease severity}


504 To further investigate the trajectory of cellular and humoral adaptive immune responses during SARS-

505 CoV-2 infection and relationship with disease severity, we performed integrative analysis on aggregated 506 immunological and clinical data from 433 samples obtained from 86 donors (12 asymptomatic, 66 mild,

5078 severe) on 6 different timepoints (Fig. 6A). We investigated the trajectory of immune responses after

508 SARS-CoV-2 infection and determined whether samples obtained from individuals with asymptomatic 509 infection are more similar to samples obtained at later timepoints after infection in the individuals with 510 mild, symptomatic disease. A t-distributed stochastic neighbour embedding (t-SNE) representation of 511 integrated data revealed heterogeneity of immune responses in infected individuals, irrespective of days 512 post symptom onset when these samples were collected (Fig. 6B, left pane/). Majority of samples were 513 separated between asymptomatic and mild individuals, while there was an overlap in similarity between 514 individuals with mild and more severe disease (Fig. 6B, right pane). To further delineate differences in 515 clinical and immunological parameters of SARS-CoV-2 infected individuals, we performed clustering 516 analysis on the resulting t-SNE representations (Fig. 6C) and compared expression of 16 clinical and 51749 immunological parameters to identify each of three clusters (Fig. 6D). This approach identified 518 heterogeneity within the SARS-CoV-2 positive individuals with mild disease clustered in two groups 519 (Fig. 6C and 6D, clusters 1 and 2). In cluster 1, the majority of samples displayed increased antibody 520 and $\mathrm{T}$ cell responses in comparison to other clusters, and some individuals with mild infection that 521 showed clinical and immunological similarity to severe COVID-19 patients (Fig. 6C and 6D, cluster 1). 522 In contrast, cluster 2 contained individuals with lower overall antibody and T cell responses and all were 523 from individuals with mild disease (Fig. 6C and 6D, cluster 2). Clinical parameters were driving a major 524 separation between asymptomatic SARS-CoV-2 positive individuals from those with mild or sever 525 disease (Fig. 6D, cluster 3).

526

527 To gain an insight into immunological differences between individuals with asymptomatic and mild 528 infection, we performed principal component analysis (PCA) on dataset containing only immunological 529 parameters. The immunological parameters alone could explain $38.6 \%$ of variance between SARS530 CoV-2 positive individuals, while separation was not driven by the disease severity (Fig. 6E). 531 Comparable to t-SNE analysis, samples from individuals with mild disease were separated into three 532 major groups having distinct immunophenotype (immunophenotypic group 1) (Fig. 6E, lower right 533 quadrant) or sharing immunological similarity with samples from individuals with severe 534 (immunophenotypic group 2) (Fig. 6E, upper right quadrant) or asymptomatic disease 535 (immunophenotypic group 3) (Fig. 6E, center). To reveal which parameters are driving the separation, 536 we visualized relationship between variables using correlation plot (Fig. 6F). T cell parameters were 537 driving the separation of immunophenotypic group 1, while antibody responses separated 538 immunophenotypic group 2 (Fig. 6F). The most important variables in explaining the variability between 539 SARS-CoV-2 positive individuals in immunophenotypic group 1 were total IFN-y ELISpot T cells, S1 540 and S2-stimulated IFN-y ELISpot T cells, and anti-S IgG, anti-RBD IgG, ADCD, S-IgG from OC43 and 541 HcoV-HKU1 in immunophenotypic group 2 that were correlated with principal components 1 and 2 542 (PC1-PC2) (Fig. 6G and 6H). The correlation plot revealed positive correlation between antibody 543 responses, and negative correlation between $T$ cell responses with the time when samples were 
544 obtained (Fig. 6F). To further examine these associations between immunological parameters, we

545 performed correlation analysis, which confirmed strong positive correlation between antibody and T 546 cells responses (Fig. 6I). The antibodies directed against N, S and RBD from SARS-CoV-2, were 547 positively correlated with antibody functionality, such as pseudoneutralising capacity and ADCD, ADNP 548 and ADMP, and positively correlated with IFN- $\gamma$ ELISpot T cell responses against S1, S2 and N (Fig. 549 6I). The antibody responses to $S$ protein from other circulating coronaviruses, such as SARS-CoV-1, 550 MERS, HcoV-HKU1, 229e and OC43 were also contained in this cluster being positively correlated with 551 antibody and T cell responses (Fig. 6I). This cluster was negatively correlated with time, confirming the 552 observations from primary analysis (Fig. 6I). Notably, there was a negative correlation between NL63 553 S antibodies and S and RBD SARS-CoV-2 specific antibodies (Fig. 6I). There were other apparent 554 relationships in two other clusters identified, that were not associated with time, including positive 555 correlation between proliferating $T$ cells stimulated with different SARS-CoV-2-specific peptides, and 556 positive correlation between ADNKA and S-IgA and S-lgG1, while negative correlation with S-IgM (Fig. 557 6I).

558 The integrative analysis revealed three distinct immunophenotypic groups of SARS-CoV-2 infected 559 individuals strongly connected to cellular and humoral immune profiling beyond the disease severity 560 and clinical parameters.

561

562 Identifying an early immunological signature associated with a durable immune response to 563 SARS-CoV-2

564 To elucidate an early immunological signature that could predict whether an individual will mount a 565 durable and protective immunity against SARS-CoV-2 6 months after infection, we stratified SARS566 CoV-2 infected individuals into high and low responders, based on the seropositivity status (N IgG titres $567>=1.4$ ), which has recently been identified as a correlate of protection ${ }^{35}$. We then asked whether the 568 components of cellular or humoral immunity within one month of infection (28 days pso) were predictive 569 of the ability of individuals to develop protective immunity against SARS-CoV-2 (6 months pso). First, 570 using an unsupervised machine learning approach, i.e., hierarchical clustering of integrated 571 immunological data on day 28 pso, we identified two groups of SARS-CoV-2 infected individuals based 572 on the response status 6 months pso (Fig. 7A). While the majority of SARS-CoV-2 infected individuals 573 with mild disease would mount protective immunity 6 months pso and become high responders, there 574 was a proportion of individuals with mild disease that failed to mount durable and protective immunity 575 (low responders) (Fig. 7A). The majority of individuals with asymptomatic infection were low 576 responders. High responders mounted stronger antibody responses, in particular N-lgG and pseudo577 neutralising antibodies, and overall, stronger $T$ cell responses, including IFN- $\mathrm{Y}$-positive and proliferating 578 T cells, than low responders 28 days pso (Fig. 7A). Antibody responses to spike protein from 229e and 579 NL63, B cell ELISpot and ADNKA were increased in low responders early after SARS-CoV-2 infection in comparison to high responders (Fig. 7A).

582 To further define the immunological features that can distinguish individuals with durable and protective 583 immunity and predict if the individual is on the trajectory to become a high or low responder, we used 
the SIMON supervised machine learning approach 28,29 . We generated 30 resamples and tested 3,565 models using 172 machine learning algorithms (Materials and methods). The best performing model built using Sparse Partial Least Squares (sPLS) algorithm (train AUROC: 0.95 (Cl 0.5-1) and test AUROC: 1) used only 8 out of 49 measured parameters on day 28 pso to predict if the individual will become high or low responder 6 months pso (Fig. 7B). The features that were contributing the most to this model included antibody responses to $\mathrm{N}$ and $\mathrm{S}, \mathrm{ADCD}$ and pseudo-neutralising antibodies to SARS-CoV-2, and T cell IFN-y ELISpot (S1/S2, M and total positive T cells) which were significantly increased in high responders 28 days pso compared to low responders (Fig. 7C and 7D). Together, these data indicate that early generation of antibodies with high binding, neutralising and effector function, and functional $T$ cell responses following infection can predict the responsiveness potential, i.e., protection and duration of SARS-CoV-2 immunity of the individual. Additionally, these findings suggest that a coordinated action of both $\mathrm{T}$ and $\mathrm{B}$ cells early after infection is required for establishment of durable and protective immunity.

The generation of durable and functional humoral and cellular immunity in a proportion of SARS-CoV2 infected individuals (high responders) may provide protection against re-infection, including also against variants of concern (VOCs). Thus, we assessed the neutralising antibody responses in high and low responders against the infecting (Victoria) strain and against variants B.1.1.7 and B.1.351 (Fig. 7E). Individuals with durable and protective SARS-CoV2 immunity shown high neutralisation antibody titres against wild-type circulating SARS-CoV-2 (Victoria) strain, and against two novel variants, including B.1.1.7 (alpha) and B.1.351 (beta) (Fig. 7E). High responders had significantly higher neutralising antibody titres against B.1.1.7 alpha variant one-month pso, and these higher neutralising antibodies were preserved 6 months pso (Fig. 7E).

607

Altogether, these data suggest that generation of immunity to SARS-COV-2 shows distinct trajectories following early priming, and early antibody responses are important to mediate protective and durable immunity that can also provide protection against novel variants.

611

\section{Discussion}

613 Key questions on the trajectory of the SARS-CoV-2 specific immune response to natural infection, and

614 the maintenance of immune memory remain highly relevant even as highly effective vaccines are being 615 rolled out worldwide. Firstly, even with high availability of vaccines there will always be a pool of 616 unvaccinated people due to vaccine hesitancy or access difficulties, and this will include people who 617 have had natural infection. Secondly, as of June 2021 only $12 \%$ of the world's population is estimated 618 to have received at least one dose of vaccine ${ }^{36}$, so for much of the immunity globally is from natural 619 infection, which remains a cornerstone of population-level immunity. Thirdly, measuring immune 620 responses to antigens not included in spike-containing vaccines are used as biomarkers of previous 621 SARS-CoV-2 infection and as such are widely used to stratify immune responses to vaccination, since 622 prior SARS-CoV-2 is known to enhance vaccine responsiveness ${ }^{37,38}$. Finally, understanding how the 623 early immune response translates into lasting immunity towards emerging variants of concern is crucial 
624 to accelerate predictions of population risk and to drive policy. In this manuscript, we characterise the

625 magnitude, function and maintenance of humoral and cellular $T$ and $B$ cell immunity, and the 626 relationship between clinical and multi parametric immune data. We then evaluate the ability of 627 antibodies to neutralise live SARS-CoV-2 virus 6 months after primary infection to variants of concern 628 and provide insight into the early predictors of durable neutralising antibody after natural infection.

629

630 Compatible with other studies $12,39,40,41$, our data shows a peak of anti-NP and anti-S binding antibody 631 (IgG) magnitude 28 days after onset of symptoms, with anti-NP responses declining over the next five 632 months, although these responses remain above the threshold of detection in the majority. In contrast, 633 anti-S IgG responses were well maintained, in keeping with the reported longer half-life for decay of 634 anti-S IgG responses compared with anti-NP $\lg G$ responses ${ }^{12}$, along with maintenance of $B$ cell 635 memory. Neutralisation measured by a pseudo-neutralisation assay showed a decline over time but 636 was generally maintained six months following infection. High levels of neutralisation were seen earlier 637 post symptom onset (from 7 days) compared with the IgG binding assays, which may represent 638 contributions from $\operatorname{lgM}{ }^{42}$ and $\operatorname{lgA}{ }^{43}$. Some of the observed decline in neutralising antibodies over time 639 may represent a threshold effect - NAb are a subset of total IgG such that gradual declines over time 640 are first measurable in NAb, but biologically important neutralisation may still occur below the detection 641 threshold. Fc-mediated functionality including antibody dependent NK activation, phagocytosis and 642 complement deposition was maintained over the 6 months duration which may make an important 643 contribution to protective immunity and was significantly associated with increasing disease severity.

644

645 Taken together, B cell polyfunctionality was lower in those with asymptomatic infection, compared with 646 those with mild disease early after infection (day 28), though by 6 months the profiles between the 647 cohorts looked similar. The most notable changes were a reduction in IgM spike responses but a relative 648 maintenance of IgG3 spike responses in the mild cohort that was not seen in the asymptomatic cohort.

649

650 Previous studies have shown that early distinct antigenic targets and qualitative features of SARS-CoV-

651 2-specific antibodies are associated with disease trajectory 44, 45, whilst multifunctional antibody 652 responses, and particularly $A D C D$ and $A D N P$, following adoptive transfer of IgG from convalescent 653 rhesus macaques have been shown to contribute to protection from SARS-CoV-2 challenge ${ }^{46}$. 654 Furthermore, vaccine-induced Fc-mediated polyfunctionality has been observed following 655 administration of efficacious vaccines in both macaque and human studies 31,47 . While the capacity of 656 Fc receptor binding appears to be lower in convalescent individuals against VOCs, evidence is 657 emerging of maintenance of vaccine-induced Fc-functional antibody properties against VOCs 658 supporting resilience of humoral immunity against VOCs independent of neutralisation ${ }^{48}$.

660 In evaluating SARS-CoV-2 specific effector T cell responses over six months in an IFN-y ELISpot assay, 661 we showed that there was significant heterogeneity in the magnitude of responses between individuals 662 as previously reported $12,49,50$. The majority of people showed robust $T$ cell responses in the first 28 663 days after infection, though these were significantly lower in the asymptomatic cohort. Within 3 months 

$75 \%$ and were undetectable in approximately $20 \%$. We used a flow cytometry based 7 -day proliferation assay to assess memory $T$ responses of both CD4+ and CD8+ T cell subsets to show a dominant CD4+

667 T cell subset response. Although memory proliferative responses have been shown to "mature" over 668 time, particularly following vaccination ${ }^{51,52}$, we show that proliferative responses (both CD4 and CD8), 669 targeting Spike, M, and NP decline markedly between day 28 and day 180. ICS analysis showed that 670 CD4+ $T$ cells were the dominant subset targeting S1, S2 and M antigens, whilst NP were targeted by 671 both CD4+ and CD8+ T cells, and NSP3B was targeted by CD8+ T cells. Polyfunctional T cells, 672 producing multiple cytokines, were generated at day 28 , and although the magnitude of the response 673 declined, polyfunctionality was generally retained out to 6 months.

674

675 In our study we show that symptomatic infection is associated with more robust cellular and humoral 676 immune responses compared to the asymptomatic group early after PCR+ confirmed infection. An 677 association between asymptomatic infection and lower antibody responses has been previously 678 reported ${ }^{53}$, and we and others have shown a correlation between disease severity and higher levels of 679 antibody and T cell responses in early disease ${ }^{4,54}$. Similar results have been reported in other disease 680 settings including robust immune responses associated with disease severity in H1N1/09 influenza A 68155 . In contrast, a previous prospective SARS-CoV-2 screening study has observed that asymptomatic 682 infection is associated with highly functional cellular immune responses ${ }^{56}$. Either way, humoral and 683 cellular immune responses measured months after primary infection is found at low magnitude following 684 asymptomatic infection. These findings raise the possibility that people with asymptomatic SARS-CoV6852 infection may have less protective immunity months after primary infection. A limitation to our study, 686 is that the timing of infection onset in asymptomatic HCW, (even though PCR+) is not precisely defined. 687 As such, it is theoretically possible that the asymptomatic individuals in our study are later in their 688 disease course at detection, which was further explored by integrative analysis.

689

690 To elucidate the trajectory of the immune response of SARS-CoV-2 infected individuals over time and 691 identify signatures associated with the maintenance of protective immunity, we performed an integrative 692 analysis in the cohort of 86 individuals on all 433 samples. The results of the integrative analysis led to 693 several key findings. First is the identification of immunophenotypic groups of SARS-CoV-2 infected 694 individuals beyond disease time course and disease severity. By integrating over 70 immune 695 parameters with clinical data, disease severity and temporal changes, we generated a computational 696 model using t-SNE embedding algorithm that coupled immunological phenotypes of each individual 697 with the disease severity and other clinical parameters. The t-SNE representation of integrated data 698 revealed minimal clustering by time point, suggesting that heterogeneity of the immune response during 699 the SARS-CoV-2 infection is independent of the time course during the infection. While some of the 700 individuals with asymptomatic infection may be later in their disease course at detection, the majority 701 did not cluster with the samples obtained from individuals with mild or severe infection at later timepoints 702 after the infection. The major separation of individuals with asymptomatic disease was driven by clinical 703 parameters, while the mild cohort clustered into 2 immunophenotypic groups (not driven by clinical 
parameters), one of which shared phenotype with the severe disease cohort. The PCA analysis provided further support for the heterogeneity of the immune responses in the SARS-CoV-2 infected individuals with mild disease and separation into three immunophenotypic groups, confirming that $38.6 \%$ of variance between individuals was explained by the immunological data. The results suggested that immunophenotypic group 1, exhibiting robust binding (anti- $\mathrm{N}$ and anti-S) and functional (pseudoneutralising and ADCD/ADMP) antibody responses and memory $B$ cell involvement, shared similarity with individuals with severe disease, while immunophenotypic group 2 composed of functional

711 IFN-y T cell responses represented an unique proportion of individuals with mild disease, early in the 712 course of the disease (as indicated by negative correlation with time when samples were acquired). 713 The third immunophenotypic group - defined by the lower overall antibody and $T$ cell responses 714 shared similarities with the asymptomatic cohort, suggesting that some individuals may fail to develop 715 robust antibody and T cell responses despite having mild infection. These results support the magnitude 716 of the immune response being determined by factors beyond disease severity, including viral factors 717 and the individual's immunocompetence. Using correlation analyses, we observed a positive 718 association between spike and nucleocapsid T cell and antibody responses (both decreased with time, 719 confirming the primary analysis) and cross-reactivity to other coronaviruses which correlated with spike 720 and nucleocapsid T cell and antibody responses (NL63 is negatively correlated and OC43 is positively 721 correlated), substantiating the findings that immunity may be defined by immunocompetence and previous exposure to circulating coronaviruses.

724 To further delineate this observation, we performed integrative analysis using baseline parameters only 725 (measured on day 28 after infection), and this led to the second key finding - identification of an early 726 immunological signature that is associated with durable and protective SARS-CoV-2 immunity. Using 727 hierarchical clustering approach and integrated baseline cellular and humoral immune parameters, we 728 observed distinct clustering of high and low responders at this early time point. High anti-N IgG, along 729 with more robust overall T cell responses (including IFN-y ELISpot and proliferation) at baseline with a 730 low response to seasonal coronaviruses (NL63 and 229e) dominated in the high responder group, 731 whilst low responders had lower anti-N IgG and overall $T$ cell responses and had more pronounced 732 cross-reactive seasonal CoV responses (NL63 and 229e) at baseline. The final major finding was the 733 ability to predict if the individual will generate durable and protective SARS-CoV-2 immunity 6 months 734 post infection based on the early immunological signature one month after infection. With the use of 735 SIMON data mining tool and generation of more than 3,500 predictive machine learning models, we 736 identified upregulation of antibody responses (spike and NP, with pseudoneutralising and ADCD 737 functions) combined with the more robust $T$ cell responses as predictors of individuals who will generate 738 durable and protective immunity 6 months post infection (high responders). The predictive model built 739 by SIMON suggests a link between both arms of the immune response - cellular and humoral immunity 740 - with the durability of the SARS-CoV-2 protective immunity. Thus, this early immunological signature 741 may determine essential differences of the trajectory that each individual will take after SARS-CoV-2 742 infection. Importantly, the sera of the individuals who will go on to generate durable and protective 743 SARS-CoV-2 immunity (high responders) 6 months post infection, were better able to neutralise both 
the Victoria strain (the likely infection strain), and also the VOCs (B.1.1.7 - alpha and B.1.351 - beta) one month after infection, and such protective neutralising antibody responses were durable (as measured 6 months post infection). In contrast, those who were low responders 6 months after infection showed a reduction in the capacity to neutralise the Victoria strain, with a severe loss of neutralisation against both VOC - particularly B1.351.

Overall, our data reveal the highly variable range of immunity after SARS-CoV-2 infection and suggest that immune events primed during early SARS-CoV-2 infection may define the subsequent trajectories leading to the effective maintenance or loss of long-term SARS-CoV-2 protective immunity as measured by neutralising antibodies. Importantly, previous infection may not give ongoing protection against VOC months later, and people with asymptomatic infection had lower responses at all time points across many of the immune parameters we measured. Maintenance of immune memory over time is critically required for the effective neutralisation of VOC that is most likely to confer sterilising immunity, whilst other immune mechanisms including non-neutralising antibodies and $T$ cells may account for the protection against severe disease, including for VOC $57,58,59,60$. This study provides a basis for more targeted vaccination programme of previously infected individuals based on early immunological signature 28 days after infection.

761

\section{Figure Legends}

Figure 1: Longitudinal humoral immune responses in individuals with PCR confirmed SARSCoV-2 asymptomatic, mild or severe infection.

Humoral immune responses were assessed in acute and convalescent by binding antibody ELISA for total IgG specific to the (A) Nucleopcapsid and (B) Spike glycoprotein, quantification of (C) IgG memory B cells specific to the spike glycoprotein, and (D) pseudoneutralisation antibody titres. Boxplots represent the median with interquartile range, a Wilcoxon rank-sum test was used to compare between study time points. A generalised additive mixed model (GAMM) by restricted maximum likelihood right-hand plots - was used to fit the immunological measures (log10 transformed) taken at multiple study time points, using Gaussian process smooth term. Disease severity group was included in the GAMM as a linear predictor and a participant identifier was included as a random effect. See Table S1 for number of individuals evaluated per assay.

Figure 2: Antibody isotype, subclass and function in individuals with PCR confirmed SARSCoV-2 asymptomatic, mild or severe infection.

778 SARS-CoV-2 spike-specific antibody isotype and subclasses measured post-infection: (A) $\lg$ M, (B) $\lg$, 779 (C) IgG1 and (D) IgG3. Antibody function measure post-SARS-CoV-2 infection: (E) antibody-dependent 780 NK cell activation (ADNKA), (F) antibody-dependent neutrophil phagocytosis (ADNP), (G) antibodydependent monocyte phagocytosis (ADMP) and (H) antibody-dependent complement deposition (ADCD). (I) Polar plot of various antibody isotype, subclass and function data, minimum-maximum normalised. Boxplots represent the median with interquartile range, a Wilcoxon rank-sum test was used to compare between study time points. A generalised additive mixed model (GAMM) by restricted 
maximum likelihood - right-hand plots - was used to fit the immunological measures $(\log 10$ transformed) taken at multiple study time points, using Gaussian process smooth term. Disease severity group was included in the GAMM as a linear predictor and a participant identifier was included as a random effect. See Table S1 for number of individuals evaluated per assay.

Figure 3: Longitudinal specific-IgG and memory B cell responses to spike protein from nonSARS-CoV-2 coronaviruses.

(A) Meso Scale Discovery (MSD) multiplexed immunoassay (MIA) platform measurements of antibody levels to spike protein from non-SARS-CoV-2 coronaviruses. (B) Memory B cells responses to spike protein from non-SARS-CoV-2 coronaviruses. See Table $\mathrm{S} 1$ for number of individuals evaluated per assay.

796

Figure 4 Magnitude of SARS-CoV-2 specific Effector T cell Response.

(A) Ex vivo IFN-y ELISpot showing the effector T cell responses to summed SARS-CoV-2 peptide pools spanning spike, accessory and structural proteins (summed total of SARS-CoV-2 proteins tested, S1, S2, NSP3B, M, NP, ORF 3, ORF8 and the CEFT positive control peptides for T cell responses) in 78 individuals 28,90 and 180 days after mild or asymptomatic SARS-CoV-2 infection (onset of symptoms for mild cases, PCR positive test for asymptomatic participants). (B) Heatmap displaying unsupervised hierarchical clustering of the ELISpot data in (A) and disease severity (mild or asymptomatic) for the original SARS-CoV-2 diagnosis. Sfu / million PBMCs = spot forming units per million peripheral blood mononuclear cells, with background subtracted. D28, d90 and d180 = days after SARS-CoV-2 diagnosis. Grey regions on heatmap represent missing data due to insufficient cells. Plots show median with error bars indicating +/- IQR. Friedman test with Dunn's multiple comparisons test was performed.

Figure 5. Proliferative responses to SARS-CoV-2 peptide pools at 1- and 6-months post infection Proliferative responses against (A) SARS-CoV-2 proteins S1, S2, M, NP, ORF3 and ORF8 presented in CD4+ (Left hand panel) and CD8+ (Right hand panel) T cells measured at 28 and 180 days pso for

812 volunteers with mild disease or days post PCR positivity for asymptomatic disease (asymptomatic $n=$ 8138 , mild disease $n=49$ ). Kruskal Wallis $T$ test, all $P$ values are all stated on plots. $(B)$ shows unsupervised 814 hierarchical clustering showing visual representation of SARS-CoV-2 specific responses at day 28 and 815180 in both CD4+ and CD8+ T cell compartments and (C) comparative analysis of SARS-CoV-2 specific 816 CD4+ and CD8+ T cell responses at day 28 (top panel) and day 180 (bottom panel) in both 817 asymptomatic and mild groups (analysed as one group). Kruskal Wallis T test, all P values are all stated 818 on plots.

820 Figure 6. Integrative analysis of clinical and longitudinal immunological data reveals distinct 821 immunophenotypic groups of SARS-CoV-2 infected individuals. (A) Clinical study overview. (B) t822 SNE map of integrated clinical and immunological data color-coded based on timepoint or disease 823 severity. (C) Clustered t-SNE analysis. (D) Heatmap of clinical and immune parameters across three 824 identified clusters. (E) PCA plot representing integrated immunological data, grouped based on the 
825 disease severity. Percentage indicates the variance explained by the principal component (PC). (F)

826 Variable correlation plot. Positively correlated variables are grouped together, while negatively

827 correlated variables are positioned on opposite quadrants. The distance between variables and the

828 origin measures the quality of the variables on the factor map, while the colour indicated the quality of

829 representations as cos2. (G) Quality of variable representations (color-coded, cos2) and contributions

830 of variables to principal components 1 and 2 (size of the circle). (H) Top 10 variables and their

831 contribution to PC 1 and 2. (I) Correlations of immunological parameters with time component across

832 samples. Spearman's correlation coefficient (colour coded) and only significant values shown (after

833 adjusted FDR <0.05). Black boxes indicate clusters (hierarchical clustering).

834

835 Figure 7. Early signature of durable SARS-CoV2 protective immunity. (A) Hierarchical clustering

836 heatmap of immune parameters on day 28 pso, grouping by responder status 6 months pso and disease

837 severity. Results obtained using complete linkage agglomeration method, dendrogram ordered tightest

838 cluster first. (B) Integrative immunological dataset containing 3,626 datapoints (49 features and 74

839 donors) was used for SIMON analysis to predict if the individual will generate high or low anti-N antibody

840 responses 6 months pso. In total, $184 \mathrm{ML}$ algorithms were tested and 2,556 model built. ROC plot of

841 the best performing model built with the svmPoly algorithm. Train AUROC (black line) is determined

842 using 10 -fold cross-validation and test AUROC evaluated on the independent test set $(25 \%$ of the initial

843 dataset). (C) Top variables that contribute to the model and are increased in high relative to low

844 responders. (D) Frequency of selected variables on day 28pso (bars show mean with SEM). Mann-

845 Whitney test $(p<0.05)$. (E) Neutralisation assay against wild-type SARS-CoV2 (Victoria), and two novel

846 variants (B1.1.7 and B1.351) between high and low responders on two timepoints (one and 6 months

847 pso). Plots show mean with SEM. Kruskal-Wallis, with Dunn's multiple comparison test $(p<0.05)$ was

848 performed.

849

850 
852 1. Berlin DA, Gulick RM, Martinez FJ. Severe Covid-19. N Engl J Med, (2020).

2. Eyre DW, Lumley SF, O'Donnell D, Campbell M, Sims E, Lawson E, ... Walker TM. Differential occupational risks to healthcare workers from SARS-CoV-2 observed during a prospective observational study. eLife 9, e60675 (2020).

3. Fan VS, Dominitz JA, Eastment MC, Locke E, Green P, Berry K, . . loannou GN. Risk Factors for testing positive for SARS-CoV-2 in a national US healthcare system. Clin Infect Dis, (2020).

4. Peng Y, Mentzer AJ, Liu G, Yao X, Yin Z, Dong D, . . Investigators IC. Broad and strong memory CD4+ and CD8+ T cells induced by SARS-CoV-2 in UK convalescent individuals following COVID-19. Nat Immunol 21, 1336-1345 (2020).

5. Ogbe A, Kronsteiner B, Skelly DT, Pace M, Brown A, Adland E, . . Oxford Protective TClfCCT. T cell assays differentiate clinical and subclinical SARS-CoV-2 infections from crossreactive antiviral responses. Nature Communications 12, 2055 (2021).

6. Wang Z, Yang X, Zhong J, Zhou Y, Tang Z, Zhou H, . . Ran P. Exposure to SARS-CoV-2 generates T-cell memory in the absence of a detectable viral infection. Nat Commun 12, 1724 (2021).

7. Reynolds CJ, Swadling L, Gibbons JM, Pade C, Jensen MP, Diniz MO, . . Boyton RJ. Discordant neutralizing antibody and T cell responses in asymptomatic and mild SARS-CoV-2 infection. Sci Immunol 5, (2020).

8. Reuters. https://www.reuters.com/business/healthcare-pharmaceuticals/italy-give-just-onecovid-shot-some-patients-eu-struggles-with-inoculations-2021-03-04/ Accessed 13 June 2021.

9. Lumley SF, Wei J, O'Donnell D, Stoesser NE, Matthews PC, Howarth A, . . Oxford University Hospitals Staff Testing G. The duration, dynamics and determinants of SARS-CoV2 antibody responses in individual healthcare workers. Clin Infect Dis, (2021).

10. Sagar M, Reifler K, Rossi M, Miller NS, Sinha P, White LF, Mizgerd JP. Recent endemic coronavirus infection is associated with less-severe COVID-19. J Clin Invest 131, (2021).

11. Grifoni A, Weiskopf D, Ramirez SI, Mateus J, Dan JM, Moderbacher CR, . . Sette A. Targets of T Cell Responses to SARS-CoV-2 Coronavirus in Humans with COVID-19 Disease and Unexposed Individuals. Cell 181, 1489-1501 e1415 (2020).

12. Dan JM, Mateus J, Kato Y, Hastie KM, Yu ED, Faliti CE, ... Crotty S. Immunological memory to SARS-CoV-2 assessed for up to 8 months after infection. Science, (2021).

13. Ansari A, Arya R, Sachan S, Jha SN, Kalia A, Lall A, ... Gupta N. Immune Memory in Mild COVID-19 Patients and Unexposed Donors Reveals Persistent T Cell Responses After SARS-CoV-2 Infection. Front Immunol 12, 636768-636768 (2021).

14. Cromer D, Juno JA, Khoury D, Reynaldi A, Wheatley AK, Kent SJ, Davenport MP. Prospects for durable immune control of SARS-CoV-2 and prevention of reinfection. Nature reviews Immunology 21, 395-404 (2021).

15. Wheatley AK, Juno JA, Wang JJ, Selva KJ, Reynaldi A, Tan H-X, . . Kent SJ. Evolution of immune responses to SARS-CoV-2 in mild-moderate COVID-19. Nature communications 12, 1162-1162 (2021).

16. Zuo J, Dowell AC, Pearce H, Verma K, Long HM, Begum J, ... Moss P. Robust SARS-CoV2 -specific T cell immunity is maintained at 6 months following primary infection. Nat Immunol 22, 620-626 (2021). 
17. Galanti M, Shaman J. Direct observation of repeated infections with endemic coronaviruses. The Journal of Infectious Diseases, (2020).

18. Kiyuka PK, Agoti CN, Munywoki PK, Njeru R, Bett A, Otieno JR, . . Cotten M. Human Coronavirus NL63 Molecular Epidemiology and Evolutionary Patterns in Rural Coastal Kenya. The Journal of infectious diseases 217, 1728-1739 (2018).

19. Davies NG, Abbott S, Barnard RC, Jarvis Cl, Kucharski AJ, Munday JD, . . Edmunds WJ. Estimated transmissibility and impact of SARS-CoV-2 lineage B.1.1.7 in England. Science 372, (2021).

20. Supasa P, Zhou D, Dejnirattisai W, Liu C, Mentzer AJ, Ginn HM, . . . Screaton GR. Reduced neutralization of SARS-CoV-2 B.1.1.7 variant by convalescent and vaccine sera. Cell, (2021).

21. Davies NG, Jarvis Cl, Group CC-W, Edmunds WJ, Jewell NP, Diaz-Ordaz K, Keogh RH. Increased mortality in community-tested cases of SARS-CoV-2 lineage B.1.1.7. Nature 593, 270-274 (2021).

22. Zhou D, Dejnirattisai W, Supasa P, Liu C, Mentzer AJ, Ginn HM, . . Screaton GR. Evidence of escape of SARS-CoV-2 variant B.1.351 from natural and vaccine-induced sera. Cell 184, 2348-2361 e2346 (2021).

23. Tegally H, Wilkinson E, Giovanetti M, Iranzadeh A, Fonseca V, Giandhari J, . . de Oliveira T. Detection of a SARS-CoV-2 variant of concern in South Africa. Nature 592, 438-443 (2021).

24. Faria NR, Mellan TA, Whittaker C, Claro IM, Candido DDS, Mishra S, ... Sabino EC. Genomics and epidemiology of the P.1 SARS-CoV-2 lineage in Manaus, Brazil. Science 372, 815-821 (2021).

25. Dejnirattisai W, Zhou D, Supasa P, Liu C, Mentzer AJ, Ginn HM, . . Screaton GR. Antibody evasion by the P.1 strain of SARS-CoV-2. Cell.

26. Planas D, Veyer D, Baidaliuk A, Staropoli I, Guivel-Benhassine F, Rajah MM, ... Schwartz O. Reduced sensitivity of infectious SARS-CoV-2 variant B.1.617.2 to monoclonal antibodies and sera from convalescent and vaccinated individuals. bioRxiv, 2021.2005.2026.445838 (2021).

27. Skelly DT, Harding AC, Gilbert-Jaramillo J, Knight ML, Longet S, Brown A, . . James W, S, Two doses of SARS-CoV-2 vaccination induce more robust immune responses to emerging SARS-CoV-2 variants of concern than does natural infection. Research Square, (2021).

28. Tomic A, Tomic I, Rosenberg-Hasson Y, Dekker CL, Maecker HT, Davis MM. SIMON, an Automated Machine Learning System, Reveals Immune Signatures of Influenza Vaccine Responses. Journal of immunology (Baltimore, Md : 1950) 203, 749-759 (2019).

29. Tomic A, Tomic I, Waldron L, Geistlinger L, Kuhn M, Spreng RL, . . Davis MM. SIMON: Open-Source Knowledge Discovery Platform. Patterns (New York, NY) 2, 100178-100178 (2021).

30. Folegatti PM, Ewer KJ, Aley PK, Angus B, Becker S, Belij-Rammerstorfer S, . . Oxford CVTG. Safety and immunogenicity of the ChAdOx1 nCoV-19 vaccine against SARS-CoV-2: a preliminary report of a phase $1 / 2$, single-blind, randomised controlled trial. Lancet 396, 467478 (2020).

31. Barrett JR, Belij-Rammerstorfer S, Dold C, Ewer KJ, Folegatti PM, Gilbride C, . . Oxford CVTG. Phase $1 / 2$ trial of SARS-CoV-2 vaccine ChAdOx1 nCoV-19 with a booster dose induces multifunctional antibody responses. Nat Med 27, 279-288 (2021). 
32. Frey A, Di Canzio J, Zurakowski D. A statistically defined endpoint titer determination method for immunoassays. J Immunol Methods 221, 35-41 (1998).

33. Wickham H. ggplot2: Elegant Graphics for Data Analysis. Springer-Verlag New York (2016).

34. Wood S, Scheipl F. gamm4: Generalized Additive Mixed Models using 'mgcv' and 'Ime4'. R package version 0.2-6.) (2020).

35. Lumley SF, O'Donnell D, Stoesser NE, Matthews PC, Howarth A, Hatch SB, ... Oxford University Hospitals Staff Testing G. Antibody Status and Incidence of SARS-CoV-2 Infection in Health Care Workers. N Engl J Med, (2020).

36. Mathieu E, Ritchie H, Ortiz-Ospina E, Roser M, Hasell J, Appel C, . . Rodés-Guirao L. A global database of COVID-19 vaccinations. Nature Human Behaviour, (2021).

37. Krammer F, Srivastava K, Alshammary H, Amoako AA, Awawda MH, Beach KF, ... Simon V. Antibody Responses in Seropositive Persons after a Single Dose of SARS-CoV-2 mRNA Vaccine. N Engl J Med 384, 1372-1374 (2021).

38. Prendecki M, Clarke C, Brown J, Cox A, Gleeson S, Guckian M, ... Willicombe M. Effect of previous SARS-CoV-2 infection on humoral and T-cell responses to single-dose BNT162b2 vaccine. Lancet 397, 1178-1181 (2021).

39. Röltgen K, Powell AE, Wirz OF, Stevens BA, Hogan CA, Najeeb J, . . Boyd SD. Defining the features and duration of antibody responses to SARS-CoV-2 infection associated with disease severity and outcome. Science Immunology 5, eabe0240 (2020).

40. Wajnberg A, Amanat F, Firpo A, Altman DR, Bailey MJ, Mansour M, . . Cordon-Cardo C. Robust neutralizing antibodies to SARS-CoV-2 infection persist for months. Science 370, 1227 (2020).

41. Lumley SF, Wei J, O'Donnell D, Stoesser NE, Matthews PC, Howarth A, . . Oxford University Hospitals Staff Testing G. The duration, dynamics and determinants of SARS-CoV2 antibody responses in individual healthcare workers. Clinical infectious diseases : an official publication of the Infectious Diseases Society of America, ciab004 (2021).

42. Klingler J, Weiss S, Itri V, Liu X, Oguntuyo KY, Stevens C, ... Hioe CE. Role of Immunoglobulin $\mathrm{M}$ and $\mathrm{A}$ Antibodies in the Neutralization of Severe Acute Respiratory Syndrome Coronavirus 2. The Journal of infectious diseases 223, 957-970 (2021).

43. Sterlin D, Mathian A, Miyara M, Mohr A, Anna F, Claër L, .. Gorochov G. IgA dominates the early neutralizing antibody response to SARS-CoV-2. Sci Transl Med 13, eabd2223 (2021).

44. Atyeo C, Fischinger S, Zohar T, Slein MD, Burke J, Loos C, ... Alter G. Distinct Early Serological Signatures Track with SARS-CoV-2 Survival. Immunity 53, 524-532 e524 (2020).

45. Zohar T, Loos C, Fischinger S, Atyeo C, Wang C, Slein MD, ... Alter G. Compromised Humoral Functional Evolution Tracks with SARS-CoV-2 Mortality. Cell 183, 1508-1519 e1512 (2020).

46. McMahan K, Yu J, Mercado NB, Loos C, Tostanoski LH, Chandrashekar A, . . Barouch DH. Correlates of protection against SARS-CoV-2 in rhesus macaques. Nature 590, 630-634 (2021).

47. Gorman MJ, Patel N, Guebre-Xabier M, Zhu A, Atyeo C, Pullen KM, ... Alter G. Collaboration between the Fab and Fc contribute to maximal protection against SARS-CoV-2 in nonhuman primates following NVX-CoV2373 subunit vaccine with Matrix-M vaccination. bioRxiv, (2021). 
1030

48. Kaplonek P, Fischinger S, Cizmeci D, Bartsch Y, Kang J, Burke J, . . Alter G. Resilient FcEffector Functions Across SARS-CoV-2 Variants of Concern Following mRNA-1273 Vaccination.). SSRN 10.2139/ssrn.3832979 (2021).

49. Reynolds CJ, Swadling L, Gibbons JM, Pade C, Jensen MP, Diniz MO, . . Boyton RJ. Discordant neutralizing antibody and T cell responses in asymptomatic and mild SARS-CoV-2 infection. Science Immunology 5, eabf3698 (2020).

50. Zuo J, Dowell AC, Pearce H, Verma K, Long HM, Begum J, ... Moss P. Robust SARS-CoV2 -specific $T$ cell immunity is maintained at 6 months following primary infection. Nature Immunology, (2021).

51. Ahlers JD, Belyakov IM. Memories that last forever: strategies for optimizing vaccine T-cell memory. Blood 115, 1678-1689 (2010).

52. Sallusto $F$, Lanzavecchia A, Araki K, Ahmed R. From vaccines to memory and back. Immunity 33, 451-463 (2010).

53. Wellinghausen N, Plonné D, Voss M, Ivanova R, Frodl R, Deininger S. SARS-CoV-2-IgG response is different in COVID-19 outpatients and asymptomatic contact persons. $J$ Clin Virol 130, 104542 (2020).

54. Lynch KL, Whitman JD, Lacanienta NP, Beckerdite EW, Kastner SA, Shy BR, . . Wu AHB. Magnitude and Kinetics of Anti-Severe Acute Respiratory Syndrome Coronavirus 2 Antibody Responses and Their Relationship to Disease Severity. Clinical infectious diseases : an official publication of the Infectious Diseases Society of America 72, 301-308 (2021).

55. Zhao Y, Zhang YH, Denney L, Young D, Powell TJ, Peng YC, ... Dong T. High levels of virus-specific CD4+ T cells predict severe pandemic influenza A virus infection. Am J Respir Crit Care Med 186, 1292-1297 (2012).

56. Le Bert N, Clapham HE, Tan AT, Chia WN, Tham CYL, Lim JM, . . Tam CC. Highly functional virus-specific cellular immune response in asymptomatic SARS-CoV-2 infection. $J$ Exp Med 218, (2021).

57. Vasileiou E, Simpson CR, Shi T, Kerr S, Agrawal U, Akbari A, . . Sheikh A. Interim findings from first-dose mass COVID-19 vaccination roll-out and COVID-19 hospital admissions in Scotland: a national prospective cohort study. Lancet (London, England) 397, 1646-1657 (2021).

58. Lopez Bernal J, Andrews N, Gower C, Robertson C, Stowe J, Tessier E, ... Ramsay M. Effectiveness of the Pfizer-BioNTech and Oxford-AstraZeneca vaccines on covid-19 related symptoms, hospital admissions, and mortality in older adults in England: test negative casecontrol study. BMJ 373, n1088 (2021).

59. Sadoff J, Gray G, Vandebosch A, Cárdenas V, Shukarev G, Grinsztejn B, . . Douoguih M. Safety and Efficacy of Single-Dose Ad26.COV2.S Vaccine against Covid-19. N Engl J Med 384, 2187-2201 (2021).

60. Fischer RJ, van Doremalen N, Adney DR, Yinda CK, Port JR, Holbrook MG, . . Munster VJ. ChAdOx1 nCoV-19 (AZD1222) protects hamsters against SARS-CoV-2 B.1.351 and B.1.1.7 disease. Preprint at https://doi.org/10.1101/2021.03.11.435000 (2021). 


\section{Figures}
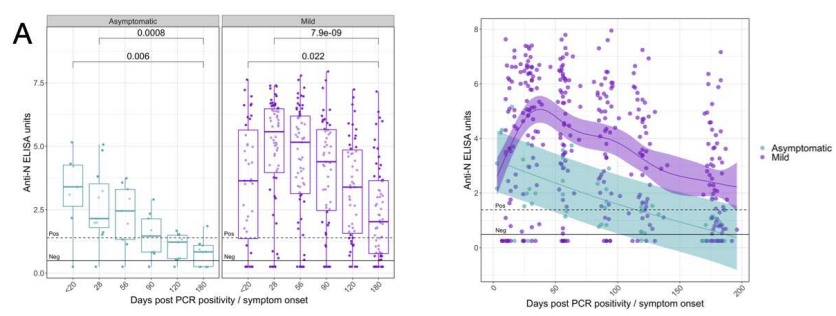

B
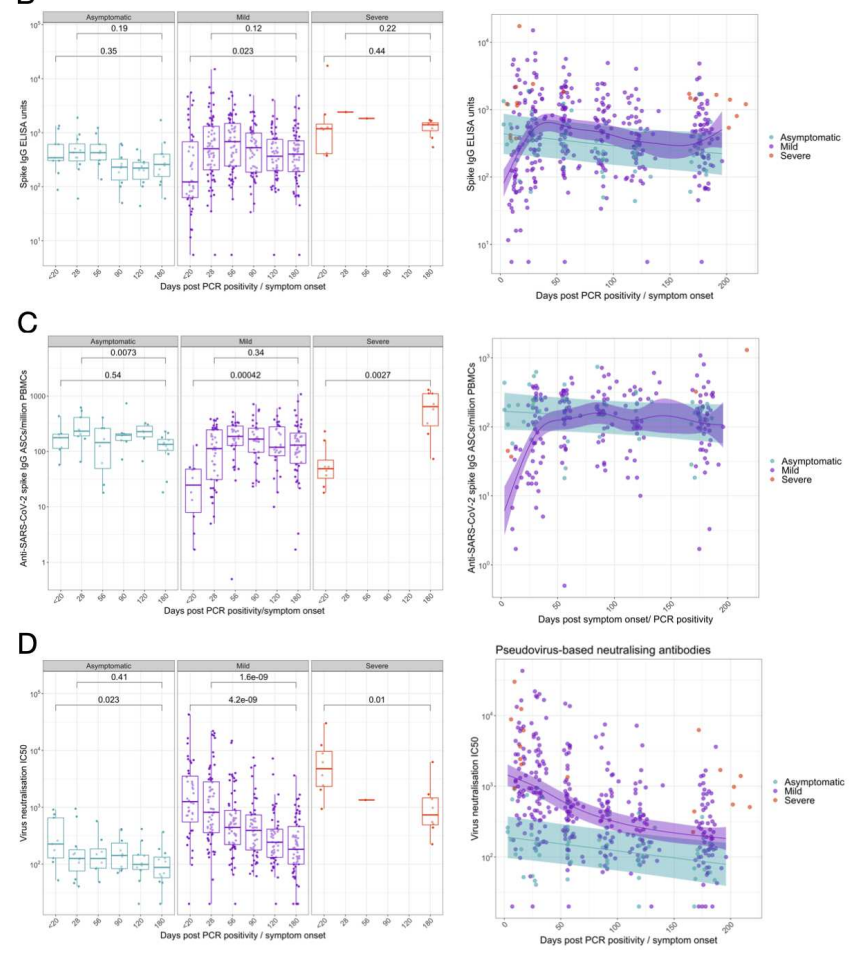

Figure 1

\section{Figure 1}

Figure 1: Longitudinal humoral immune responses in individuals with PCR confirmed SARS-CoV-2 asymptomatic, mild or severe infection. Humoral immune responses were assessed in acute and convalescent by binding antibody ELISA for total IgG specific to the (A) Nucleopcapsid and (B) Spike 
glycoprotein, quantification of (C) IgG memory B cells specific to the spike glycoprotein, and (D) pseudoneutralisation antibody titres. Boxplots represent the median with interquartile range, a Wilcoxon rank-sum test was used to compare between study time points. A generalised additive mixed model (GAMM) by restricted maximum likelihood - right-hand plots - was used to fit the immunological measures (log10 transformed) taken at multiple study time points, using Gaussian process smooth term. Disease severity group was included in the GAMM as a linear predictor and a participant identifier was included as a random effect.
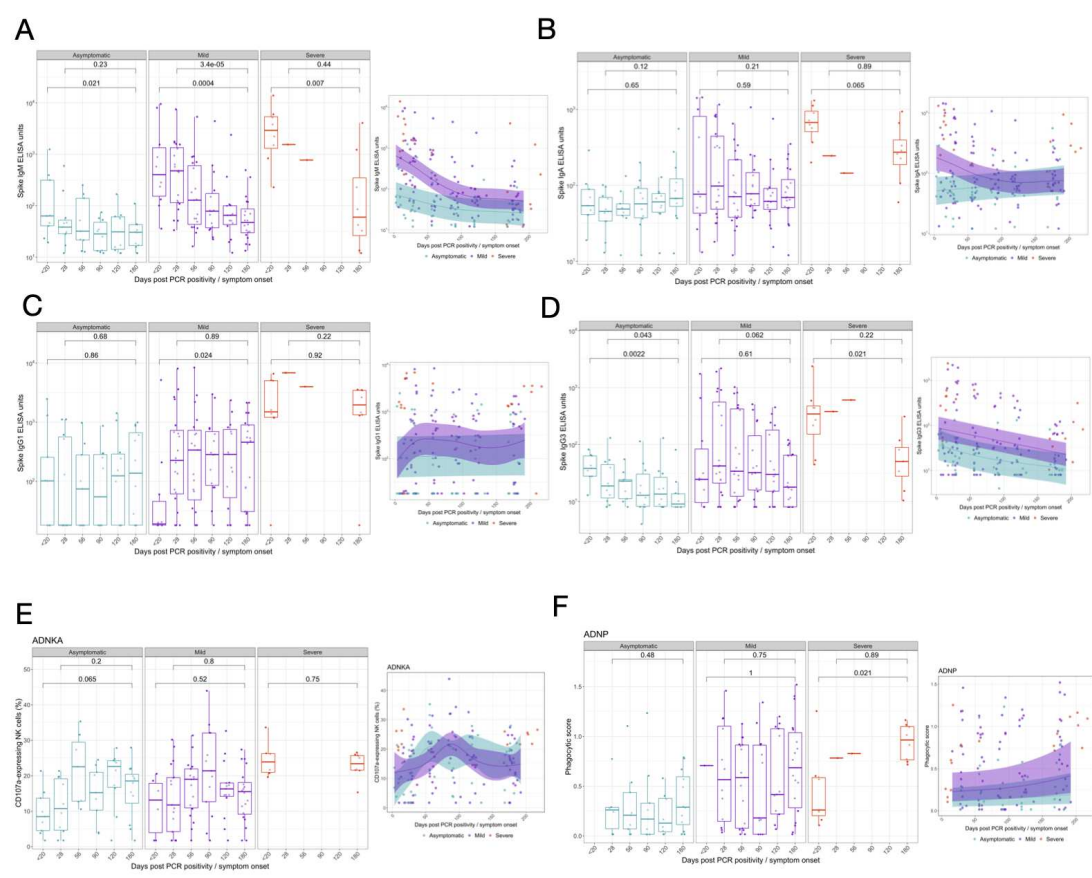

F
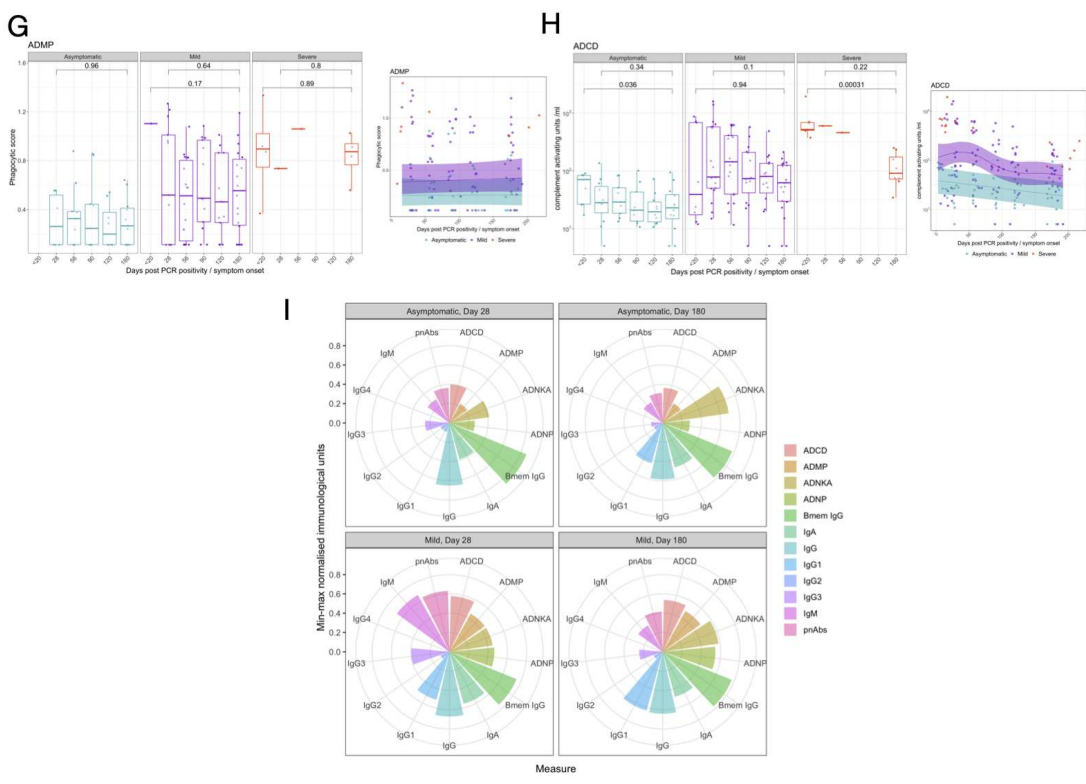

Figure 2 


\section{Figure 2}

Figure 2: Antibody isotype, subclass and function in individuals with PCR confirmed SARS-CoV-2 asymptomatic, mild or severe infection. SARS-CoV-2 spike-specific antibody isotype and subclasses measured post-infection: (A) IgM, (B) IgA, (C) IgG1 and (D) IgG3. Antibody function measure post-SARSCoV-2 infection: (E) antibody-dependent NK cell activation (ADNKA), (F) antibody-dependent neutrophil phagocytosis (ADNP), (G) antibody-dependent monocyte phagocytosis (ADMP) and (H) antibodydependent complement deposition (ADCD). (I) Polar plot of various antibody isotype, subclass and function data, minimum-maximum normalised. Boxplots represent the median with interquartile range, a Wilcoxon rank-sum test was used to compare between study time points. A generalised additive mixed model (GAMM) by restricted maximum likelihood - right-hand plots - was used to fit the immunological measures (log10 transformed) taken at multiple study time points, using Gaussian process smooth term. Disease severity group was included in the GAMM as a linear predictor and a participant identifier was included as a random effect. 
A

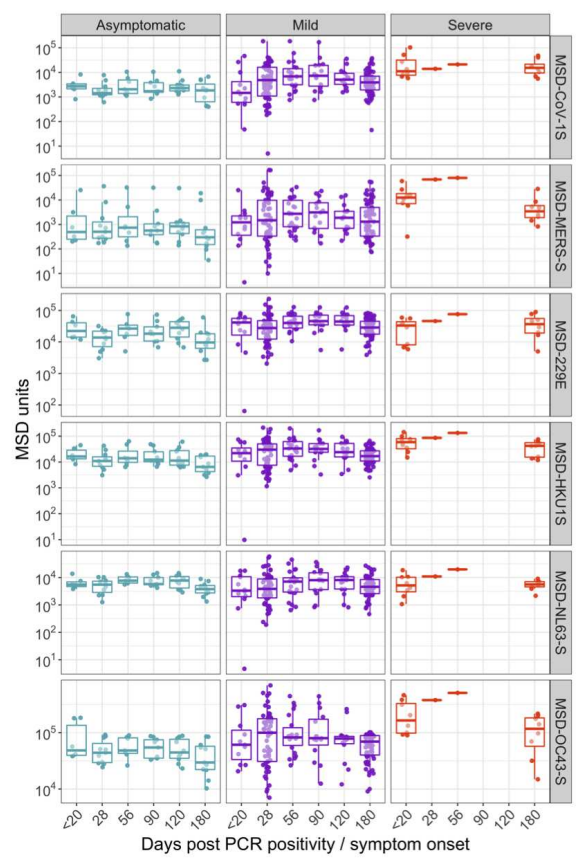

B
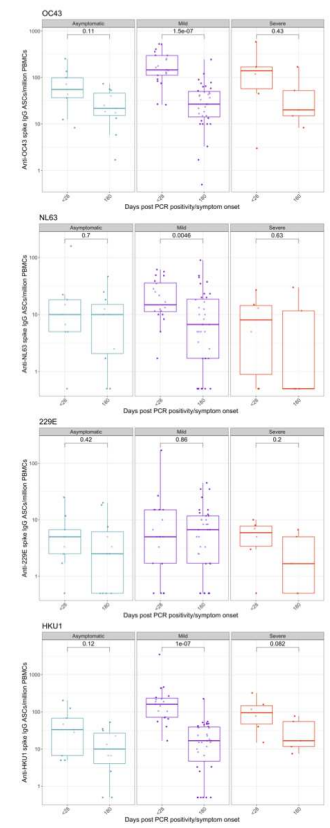

Figure 3

\section{Figure 3}

(A) Meso Scale Discovery (MSD) multiplexed immunoassay (MIA) platform measurements of antibody levels to spike protein from non-SARS-CoV-2 coronaviruses. (B) Memory B cells responses to spike protein from non-SARS-CoV-2 coronaviruses. 


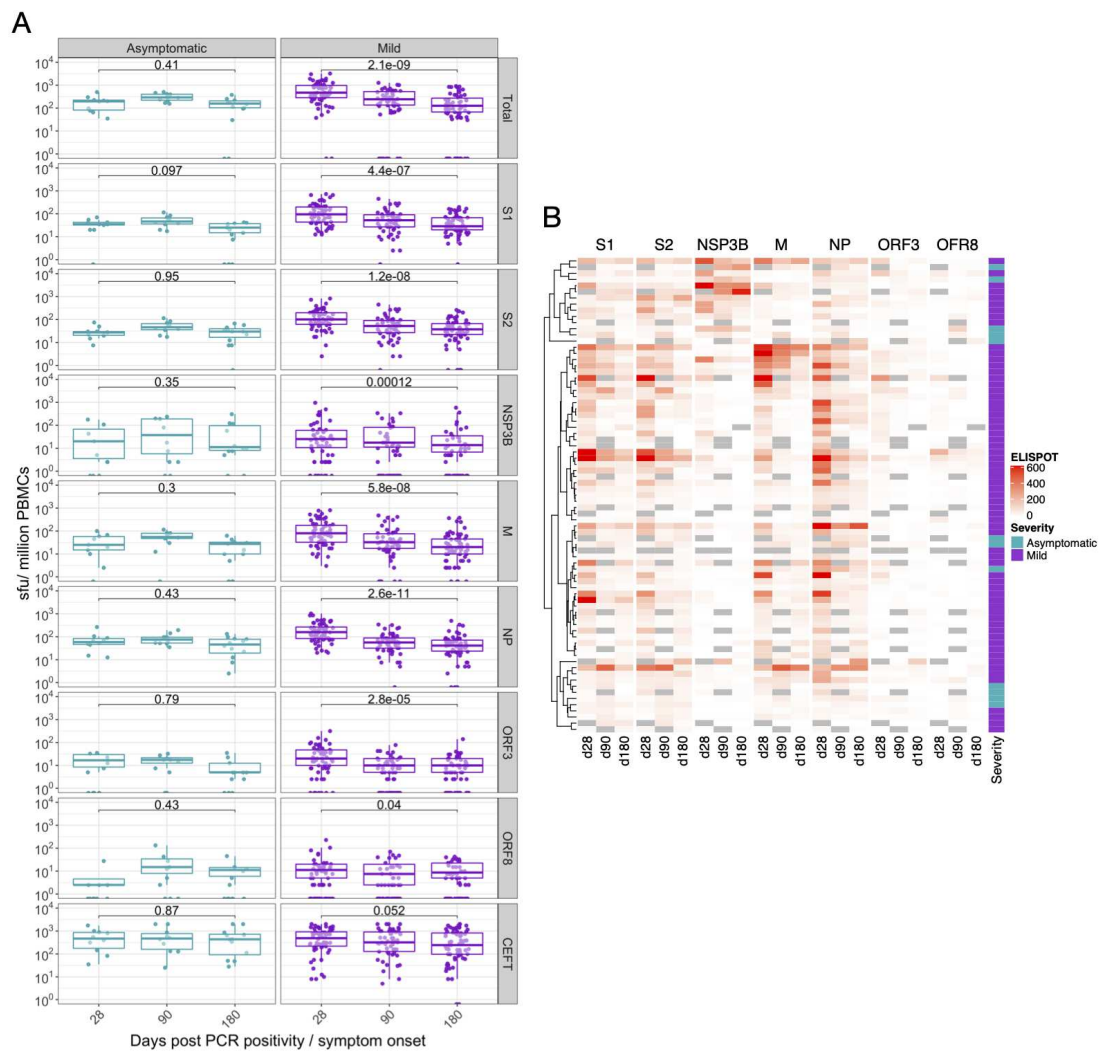

Figure 4

\section{Figure 4}

Figure 4 Magnitude of SARS-CoV-2 specific Effector T cell Response. A. Ex vivo IFN-y ELISpot showing the effector T cell responses to summed SARS-CoV-2 peptide pools spanning spike, accessory and structural proteins (M, NP, NSP3B, ORF 3, ORF8, S1, S2, summed total of SARS-CoV-2 proteins tested and the CEFT positive control peptides for T cell responses) in 78 individuals 28,90 and 180 days after SARSCoV-2 (onset of symptoms for mild cases, PCR positive test for asymptomatic participants). Heatmap 
displaying unsupervised hierarchical clustering of the ELISpot data in (A) and disease severity (mild or asymptomatic) for the original SARS-CoV-2 diagnosis. Sfu / million PBMCs = spot forming units per million peripheral blood mononuclear cells, with background subtracted. D28, d90 and d180 = days after SARS-CoV-2 diagnosis. Grey regions on heatmap represent missing data due to insufficient cells. Plots show median with error bars indicating +/- IQR. Friedman test with Dunn's multiple comparisons test was performed.

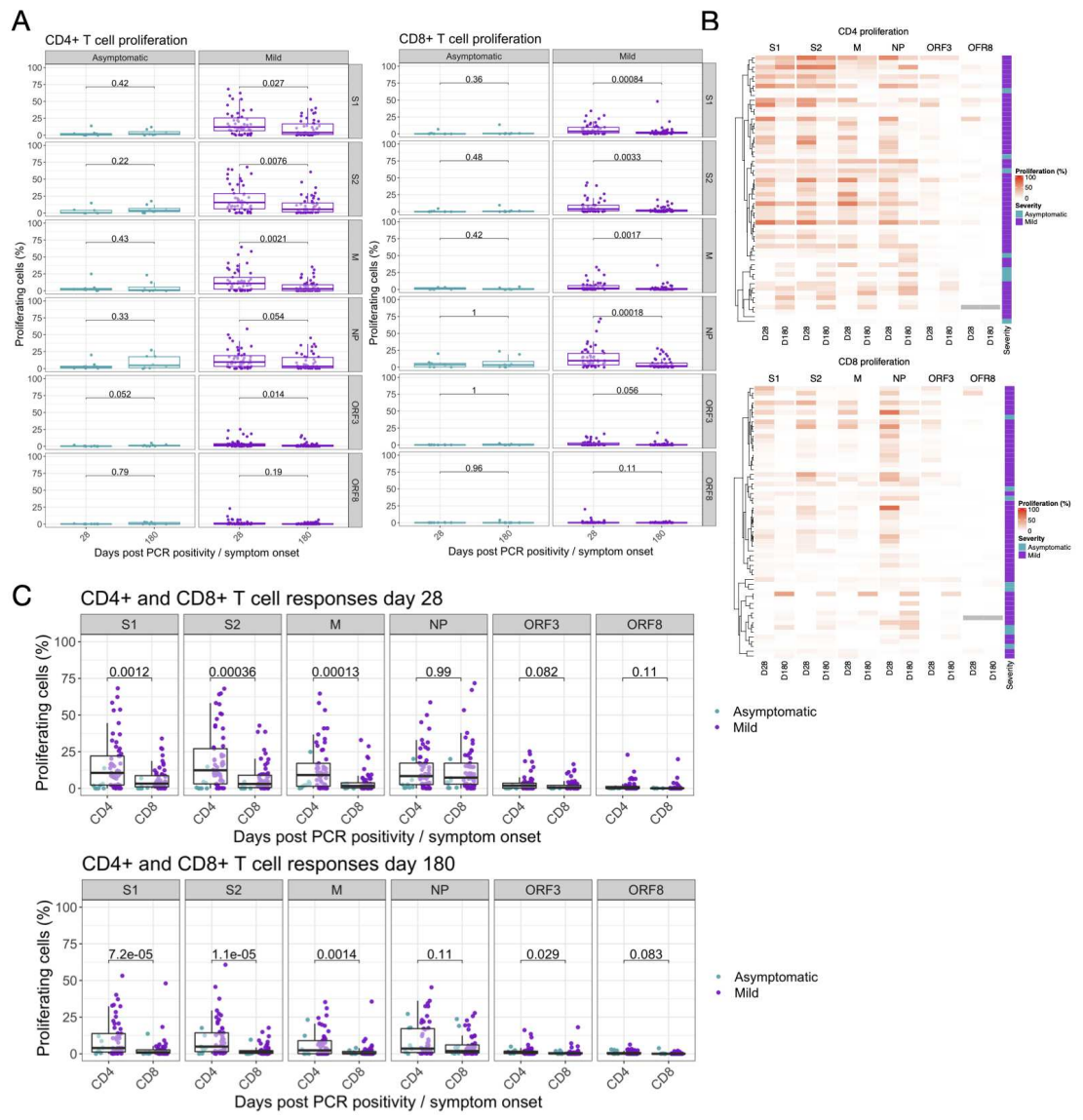

Figure 5

Figure 5 
Figure 5. Proliferative responses to SARS-CoV-2 peptide pools at 1- and 6-months post infection Proliferative responses against (A) SARS-CoV-2 proteins S1, S2, M, NP, ORF3 and ORF8 presented in CD4+ (Left hand panel) and CD8+ (Right hand panel) T cells measured at 28 and 180 days pso for volunteers with mild disease or days post PCR positivity for asymptomatic disease (asymptomatic $n=8$, mild disease $n=49$ ). Kruskal Wallis T test, all $P$ values are all stated on plots. (B) shows unsupervised hierarchical clustering showing visual representation of SARS-CoV-2 specific responses at day 28 and 180 in both CD4+ and CD8+ T cell compartments and (C) comparative analysis of SARS-CoV-2 specific CD4+ and CD8+ T cell responses at day 28 (top panel) and day 180 (bottom panel) in both asymptomatic and mild groups (analysed as one group). Kruskal Wallis T test, all P values are all stated on plots. 


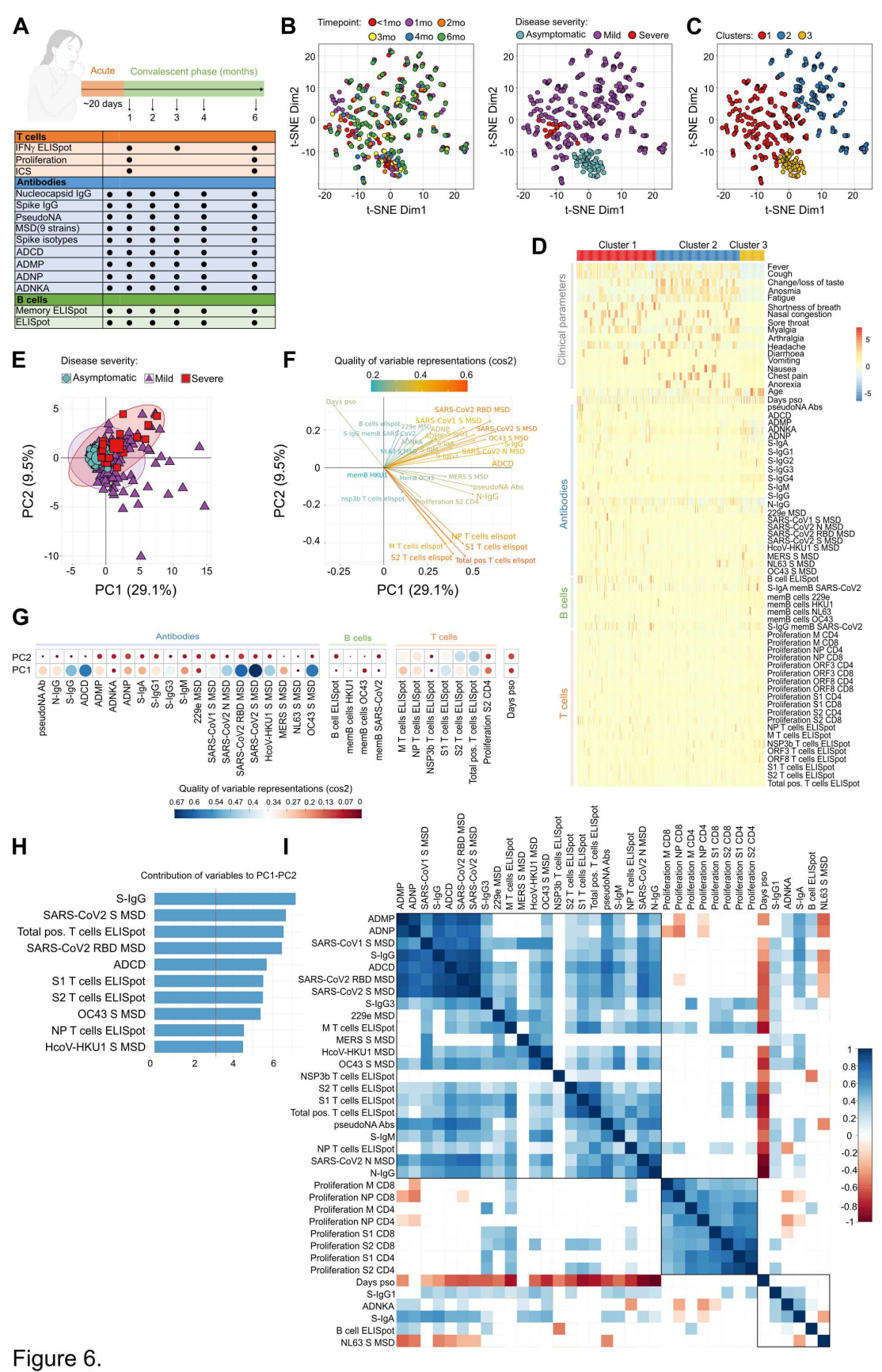

\section{Figure 6}

Figure 6. Integrative analysis of clinical and longitudinal immunological data reveals distinct immunophenotypic groups of SARS-CoV-2 infected individuals. (A) Clinical study overview. (B) t-SNE map of integrated clinical and immunological data color-coded based on timepoint or disease severity. (C) Clustered t-SNE analysis. (D) Heatmap of clinical and immune parameters across three identified clusters. (E) PCA plot representing integrated immunological data, grouped based on the disease severity. 
Percentage indicates the variance explained by the principal component (PC). (F) Variable correlation plot. Positively correlated variables are grouped together, while negatively correlated variables are positioned on opposite quadrants. The distance between variables and the origin measures the quality of the variables on the factor map, while the colour indicated the quality of representations as cos2. (G) Quality of variable representations (color-coded, $\cos 2$ ) and contributions of variables to principal components 1 and 2 (size of the circle). (H) Top 10 variables and their contribution to PC 1 and 2. (I) Correlations of immunological parameters with time component across samples. Spearman's correlation coefficient (colour coded) and only significant values shown (after adjusted FDR <0.05). Black boxes indicate clusters (hierarchical clustering). 
A

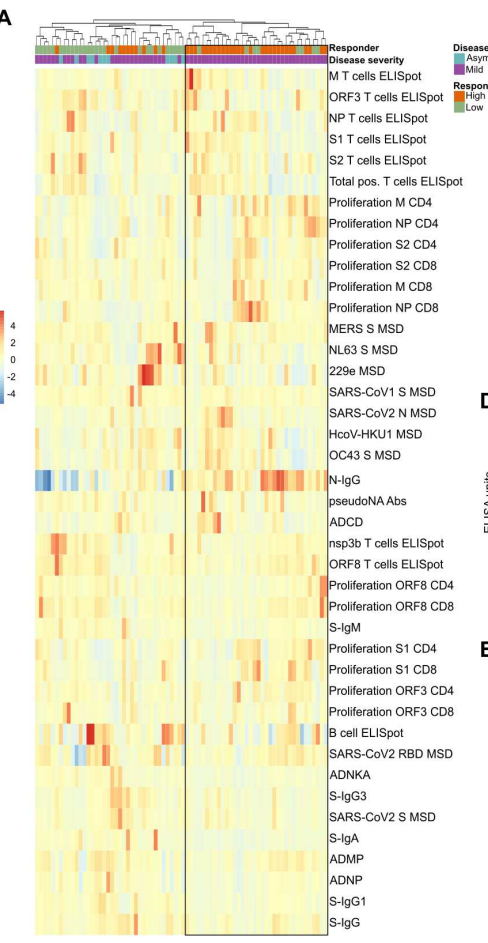

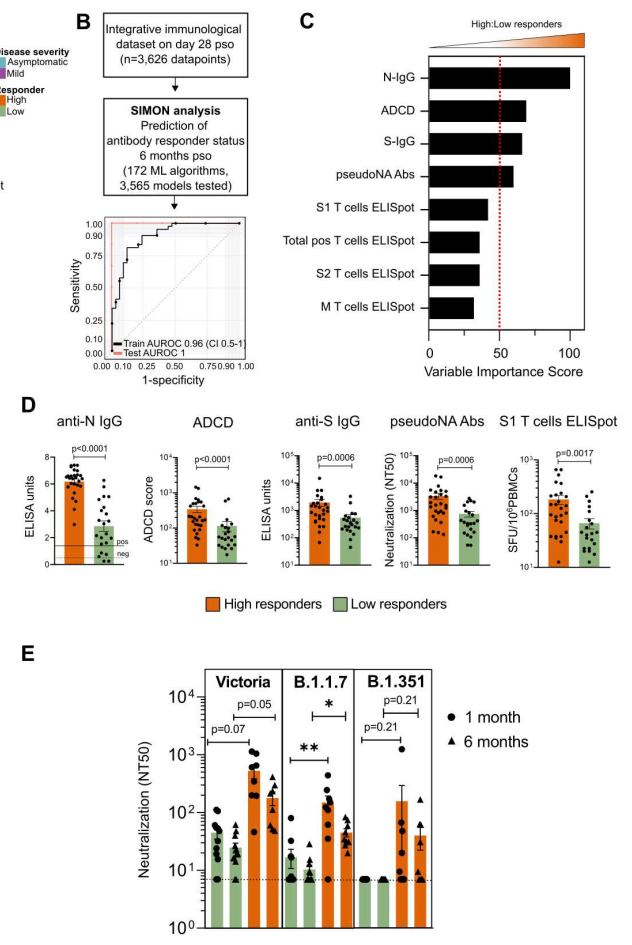

Figure 7.

\section{Figure 7}

Figure 7. Early signature of durable SARS-CoV2 protective immunity. (A) Hierarchical clustering heatmap of immune parameters on day 28 pso, grouping by responder status 6 months pso and disease severity. Results obtained using complete linkage agglomeration method, dendrogram ordered tightest cluster first. (B) Integrative immunological dataset containing 3,626 datapoints (49 features and 74 donors) was used for SIMON analysis to predict if the individual will generate high or low anti-N antibody responses 6 
months pso. In total, $184 \mathrm{ML}$ algorithms were tested and 2,556 model built. ROC plot of the best performing model built with the svmPoly algorithm. Train AUROC (black line) is determined using 10-fold cross-validation and test AUROC evaluated on the independent test set ( $25 \%$ of the initial dataset). (C) Top variables that contribute to the model and are increased in high relative to low responders. (D) Frequency of selected variables on day 28pso (bars show mean with SEM). Mann-Whitney test $(p<0.05)$. (E) Neutralisation assay against wild-type SARS-CoV2 (Victoria), and two novel variants (B1.1.7 and B1.351) between high and low responders on two timepoints (one and 6 months pso). Plots show mean with SEM. Kruskal-Wallis, with Dunn's multiple comparison test $(p<0.05)$ was performed.

\section{Supplementary Files}

This is a list of supplementary files associated with this preprint. Click to download.

- Suppfig1.tiff

- Suppfig2.tiff

- Suppfig3.tiff

- Suppfig4.tiff

- Suppfig5.tiff

- AppendixSupplementaryFiguresandAdditionalMethods.pdf 